\title{
Nonlinear normal modes, Part II: Toward a practical computation using numerical continuation techniques
}

\author{
M. Peeters, R. Viguié, G . Sérandour, G. Kerschen, J.-C. Golinval \\ Structural Dynamics Research Group, Department of Aerospace and Mechanical Engineering, University of Liège, \\ 1 Chemin des Chevreuils (B52/3), B-4000 Liège, Belgium
}

The concept of nonlinear normal modes (NNMs) is discussed in the present paper and its companion, Part I. One reason of the still limited use of NNMs in structural dynamics is that their computation is often regarded as impractical. However, when resorting to numerical algorithms, we show that the NNM computation is possible with limited implementation effort, which paves the way to a practical method for determining the NNMs of nonlinear mechanical systems. The proposed algorithm relies on two main techniques, namely a shooting procedure and a method for the continuation of NNM motions. The algorithm is demonstrated using four different mechanical systems, a weakly and a strongly nonlinear two-degree-of-freedom system, a simplified discrete model of a nonlinear bladed disk and a nonlinear cantilever beam discretized by the finite element method.

\section{Keywords:}

Nonlinear dynamics

Nonlinear normal modes

Modal analysis

Shooting method

Continuation methods of periodic motions

\section{Introduction}

Nonlinear normal modes (NNMs) offer a solid theoretical and mathematical tool for interpreting a wide class of nonlinear dynamical phenomena, yet they have a clear and simple conceptual relation to the classical linear normal modes (LNMs). The relevance of the NNMs for the structural dynamicist is addressed in Part I of this paper.

However, most structural engineers still view NNMs as a concept that is foreign to them, and they do not yet consider these nonlinear modes as a practical nonlinear analog of the LNMs. One reason supporting this statement is that most existing constructive techniques for computing NNMs are based on asymptotic approaches and rely on fairly involved mathematical developments. In this context, a significant contribution is that of Pesheck who proposed a meaningful numerical extension of the invariant manifold approach [1].

Algorithms for the numerical continuation of periodic solutions are really quite sophisticated and advanced (see, e.g., [2-5], and the AUTO and MATCONT softwares). These algorithms have been extensively used for computing the forced response and limit cycles of nonlinear dynamical systems [6-11]. Doedel and co-workers used them for the computation of periodic orbits during the free response of conservative systems [12,13].

Interestingly, there have been very few attempts to compute the periodic solutions of conservative mechanical structures (i.e., NNM motions) using numerical continuation techniques. One of the first approaches was proposed by Slater [14] who combined a shooting method with sequential continuation to solve the nonlinear boundary value problem that defines a family of NNM motions. Similar approaches were considered in Lee et al. [15] and Bajaj et al. [16]. A more 
sophisticated continuation method is the so-called asymptotic-numerical method. It is a semi-analytical technique that is based on a power series expansion of the unknowns parameterized by a control parameter. It is utilized in [17] to follow the NNM branches in conjunction with finite difference methods, following a framework similar to that of [12].

In this study, a shooting procedure is combined with the so-called pseudo-arclength continuation method for the computation of NNM motions. We show that the NNM computation is possible with limited implementation effort, which holds promise for a practical and accurate method for determining the NNMs of nonlinear vibrating structures.

This paper is organized as follows. In the next section, the two main definitions of an NNM and their fundamental properties are briefly reviewed. In Section 3, the proposed algorithm for NNM computation is presented. Its theoretical background is first recalled, and the numerical implementation is then described. Improvements are also presented for the reduction of the computational burden. The proposed algorithm is then demonstrated using four different nonlinear vibrating systems in Section 4. In Part I, the relevance of the NNMs for the dynamicist is discussed.

\section{Nonlinear normal modes (NNMs)}

A detailed description of NNMs is given in the companion paper, Part I. For completeness, the two main definitions of an NNM and their fundamental properties are briefly reviewed in this section.

\subsection{Framework and definitions}

In this study, the free response of discrete conservative mechanical systems with $n$ degrees of freedom (DOFs) is considered, assuming that continuous systems (e.g., beams, shells or plates) have been spatially discretized using the finite element method. The equations of motion are

$$
\mathbf{M} \ddot{\mathbf{x}}(t)+\mathbf{K x}(t)+\mathbf{f}_{n l}\{\mathbf{x}(t), \dot{\mathbf{x}}(t)\}=0
$$

where $\mathbf{M}$ is the mass matrix; $\mathbf{K}$ is the stiffness matrix; $\mathbf{x}, \dot{\mathbf{x}}$ and $\ddot{\mathbf{x}}$ are the displacement, velocity and acceleration vectors, respectively; $\mathbf{f}_{n l}$ is the nonlinear restoring force vector, assumed to be regular. In principle, systems with nonsmooth nonlinearities can be studied with the proposed method, but they require a special treatment [18].

There exist two main definitions of an NNM in the literature due to Rosenberg [19-21] and Shaw and Pierre [22-25]:

1. Targeting a straightforward nonlinear extension of the LNM concept, Rosenberg defined an NNM motion as a vibration in unison of the system (i.e., a synchronous periodic oscillation).

2. To provide an extension of the NNM concept to damped systems, Shaw and Pierre defined an NNM as a two-dimensional invariant manifold in phase space. Such a manifold is invariant under the flow (i.e., orbits that start out in the manifold remain in it for all time), which generalizes the invariance property of LNMs to nonlinear systems.

At first glance, Rosenberg's definition may appear restrictive in two cases. Firstly, it cannot be easily extended to nonconservative systems. However, as discussed in the companion paper, Part I, and in [15,26], the damped dynamics can often be interpreted based on the topological structure of the NNMs of the underlying conservative system. Moreover, due to the lack of knowledge of damping mechanisms, engineering design in industry is often based on the conservative system, and this even for linear vibrating structures. Secondly, in the presence of internal resonances, the NNM motion is no longer synchronous, but it is still periodic.

In the present study, an NNM motion is therefore defined as a (nonnecessarily synchronous) periodic motion of the undamped mechanical system (1). As we will show, this extended definition is particularly attractive when targeting a numerical computation of the NNMs. It enables the nonlinear modes to be effectively computed using algorithms for the continuation of periodic solutions, which are really quite sophisticated and advanced.

\subsection{Fundamental properties}

\subsubsection{Frequency-energy dependence}

One typical dynamical feature of nonlinear systems is the frequency-energy dependence of their oscillations. As a result, the modal curves and frequencies of NNMs depend on the total energy in the system. In view of this dependence, the representation of NNMs in a frequency-energy plot (FEP) is particularly convenient. An NNM motion is represented by a point in the FEP, which is drawn at a frequency corresponding to the minimal period of the periodic motion and at an energy corresponding to the conserved total energy during the motion, which is the sum of the potential and kinetic energies. A branch, represented by a solid line, is a family of NNM motions possessing the same qualitative features.

A two-degree-of-freedom (2DOF) system with a cubic stiffness is chosen. The system is depicted in Fig. 1, and its NNMs are discussed in more detail in Section 4 . The governing equations of motion are

$$
\begin{aligned}
& \ddot{x}_{1}+\left(2 x_{1}-x_{2}\right)+0.5 x_{1}^{3}=0 \\
& \ddot{x}_{2}+\left(2 x_{2}-x_{1}\right)=0
\end{aligned}
$$




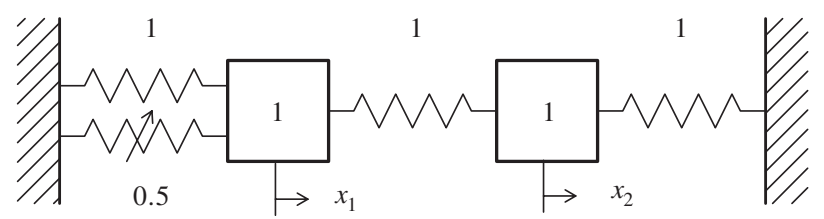

Fig. 1. Schematic representation of the 2DOF system example.

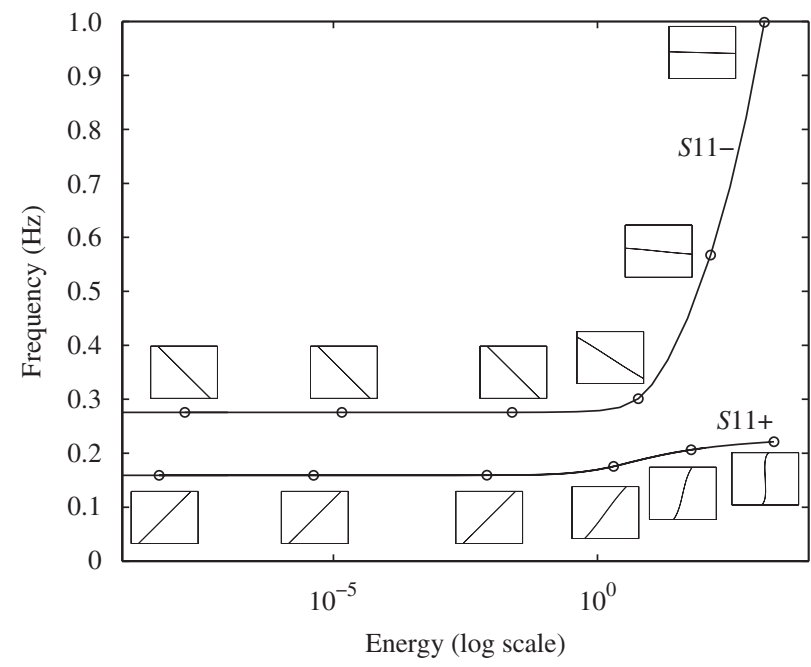

Fig. 2. Frequency-energy plot of system (2) computed with the proposed numerical method. NNM motions depicted in the configuration space are inset. The horizontal and vertical axes in these plots are the displacements of the first and second DoFs, respectively; the aspect ratio is set so that increments on the horizontal and vertical axes are equal in size to indicate whether or not the motion is localized to a particular DOF.

The underlying linear system possesses two (in-phase and out-of-phase) linear modes. The FEP of this nonlinear system, shown in Fig. 2, was obtained using the NNM computation method proposed in Section 3. The evolution of NNM motions in the configuration space (i.e., the modal curves) are inset. The backbone of the plot is formed by two branches, which represent in-phase $(S 11+)$ and out-of-phase $(S 11-)$ synchronous NNMs. They are the continuation of the corresponding LNMs. The letter $S$ refers to symmetric periodic solutions for which the displacements and velocities of the system at halfperiod are equal but with an opposite sign to those at time $t=0$. The indices in the notations are used to mention that the two masses vibrate with the same dominant frequency. The FEP clearly shows that the nonlinear modal parameters, namely the modal curves and the corresponding frequencies of oscillation, have a strong dependence on the total energy in the system.

\subsubsection{Internally resonant NNMs}

Another salient feature of NNMs is that they can undergo modal interactions through internal resonances. When carrying out the NNM computation for system (2) at higher energy levels, Fig. 3 shows that others branches of periodic solutions, termed tongues, bifurcate from the backbone branch $S 11+$. For instance, unsymmetric periodic solutions are encountered and are denoted by a letter $U$. On these tongues, denoted Snm and Unm, there exist several dominant frequency components, which results in a $n: m$ internal resonance between the in-phase and out-of-phase NNMs. These additional branches correspond to internally resonant NNM motions, as opposed to fundamental NNM motions; they have no counterpart in linear systems.

The time series and modal curves corresponding to different NNM motions of system (2) are represented in Figs. 4 and 5. Fig. 4 shows a fundamental NNM motion on $S 11+$. Three internally resonant NNM motions, namely a motion on $S 31$ and two different motions on $U 21$, are illustrated in Fig. 5. The difference between symmetric and unsymmetric NNM motions, as discussed in the previous section, is evident in this plot. It can also be observed that an NNM motion may take the form of an open or a closed curve in the configuration space. Closed orbits imply phase differences between the two oscillators of the system; i.e., their velocities do not vanish at the same time instant. Interestingly, there exist two different tongues of 2:1 internal resonance in this system, depending on whether the NNM motion is an open or closed orbit in the configuration space. These tongues are coincident and cannot be distinguished in Fig. 3. 


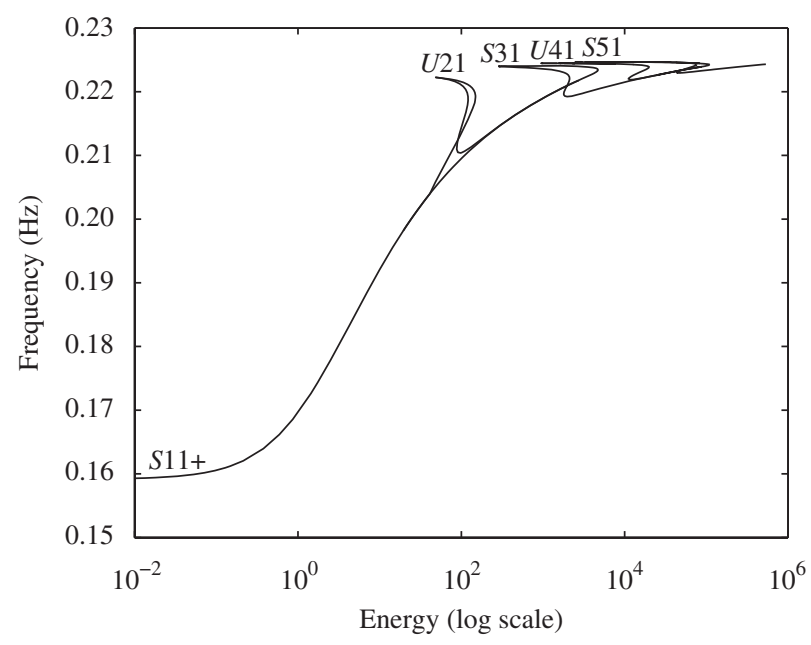

Fig. 3. $S 11+$ at higher energy levels and internally resonant NNMs (U21, S31, U41, S51).
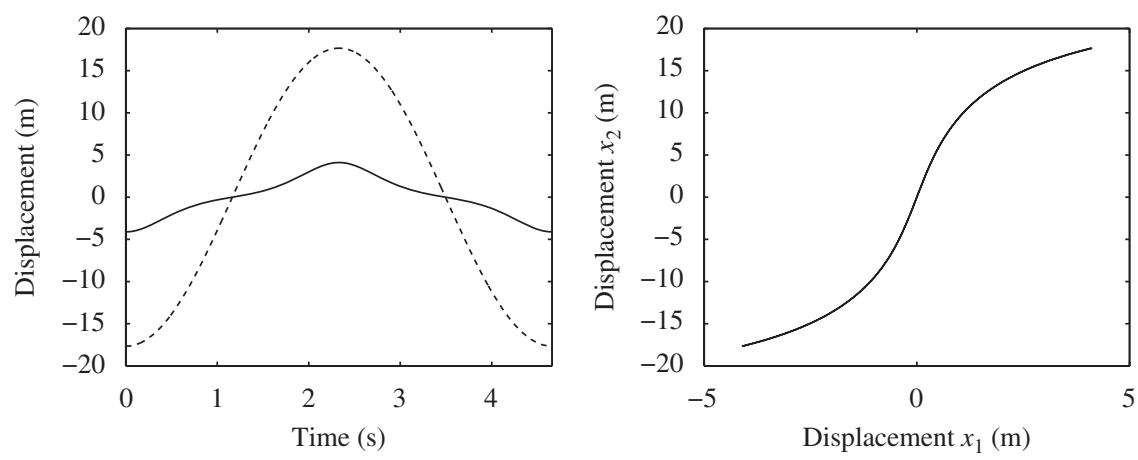

Fig. 4. Fundamental NNM motion on $S 11+\left(\right.$ Frequency $=0.213 \mathrm{~Hz}$; energy $\left.=10^{2.33} \mathrm{~J}\right)$. Left: time series $\left(-\right.$ : $x_{1}(t)$; - - -: $\left.x_{2}(t)\right)$. Right: modal curve in the configuration space.

\subsubsection{Mode bifurcations}

A third fundamental property of NNMs is that their number may exceed the number of DOFs of the system. Due to mode bifurcations, not all NNMs can be regarded as nonlinear continuation of the underlying LNMs, and these bifurcating NNMs are essentially nonlinear with no linear counterparts. Modes generated through internal resonances are one example. Another possible example corresponds to the generation of additional fundamental NNMs. This is discussed at length in [27-29].

\section{Numerical computation of NNMs}

The numerical method proposed here for the NNM computation relies on two main techniques, namely a shooting technique and the pseudo-arclength continuation method. It is summarized in Fig. 6.

\subsection{Shooting method}

The equations of motion of system (1) can be recast into state space form

$$
\dot{\mathbf{z}}=\mathbf{g}(\mathbf{z})
$$

where $\mathbf{z}=\left[\mathbf{x}^{*} \dot{\mathbf{x}}^{*}\right]^{*}$ is the $2 n$-dimensional state vector, and star denotes the transpose operation, and

$$
\left.\mathbf{g}(\mathbf{z})=\quad \begin{array}{c}
\dot{\mathbf{x}} \\
-\mathbf{M}^{-1}\left[\mathbf{K} \mathbf{x}+\mathbf{f}_{n l}(\mathbf{x}, \dot{\mathbf{x}})\right]
\end{array}\right)
$$



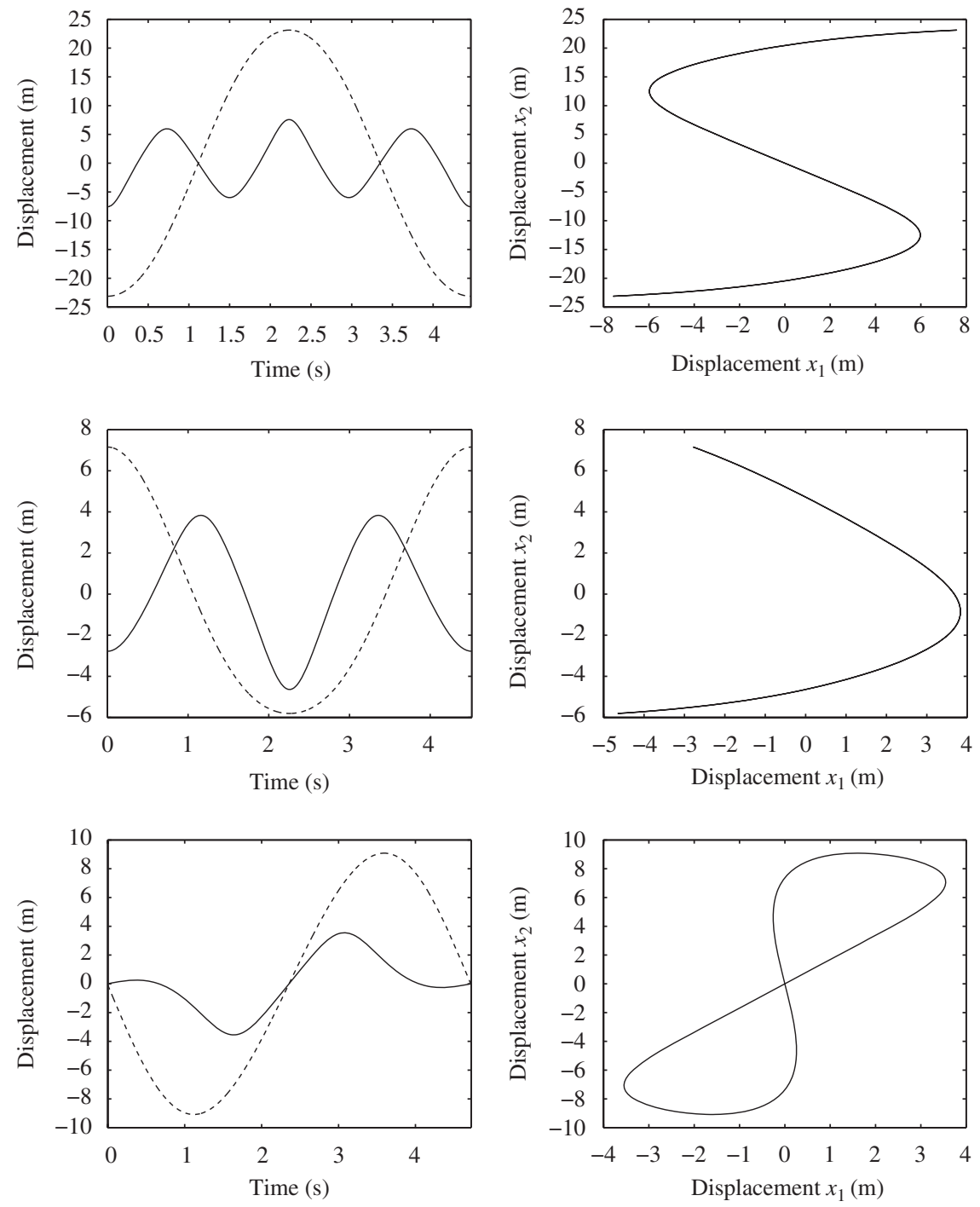

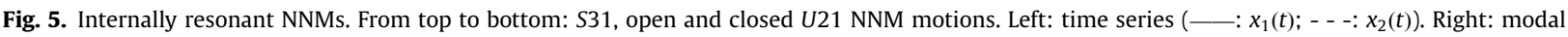
curve in the configuration space.

is the vector field. It is assumed that the mass matrix is invertible. The solution of this dynamical system for initial conditions $\mathbf{z}(0)=\mathbf{z}_{0}=\left[\mathbf{x}_{0}^{*} \dot{\mathbf{x}}_{0}^{*}\right]^{*}$ is written as $\mathbf{z}(t)=\mathbf{z}\left(t, \mathbf{z}_{0}\right)$ in order to exhibit the dependence on the initial conditions, $\mathbf{z}\left(0, \mathbf{z}_{0}\right)=\mathbf{z}_{0}$. A solution $\mathbf{z}_{p}\left(t, \mathbf{z}_{p 0}\right)$ is a periodic solution of the autonomous system (3) if $\mathbf{z}_{p}\left(t, \mathbf{z}_{p 0}\right)=\mathbf{z}_{p}\left(t+T, \mathbf{z}_{p 0}\right)$, where $T$ is the minimal period.

The NNM computation is carried out by finding the periodic solutions of the governing nonlinear equations of motion (3). In this context, the shooting method is probably the most popular numerical technique $[2,4,5,30]$. It solves numerically the two-point boundary-value problem defined by the periodicity condition

$$
\mathbf{H}\left(\mathbf{z}_{p 0}, T\right) \equiv \mathbf{z}_{p}\left(T, \mathbf{z}_{p 0}\right)-\mathbf{z}_{p 0}=\mathbf{0}
$$

$\mathbf{H}\left(\mathbf{z}_{0}, T\right)=\mathbf{z}\left(T, \mathbf{z}_{0}\right)-\mathbf{z}_{0}$ is called the shooting function and represents the difference between the initial conditions and the system response at time $T$. Unlike forced motion, the period $T$ of the free response is not known a priori.

The shooting method consists in finding, in an iterative way, the initial conditions $\mathbf{z}_{p 0}$ and the period $T$ that realized a periodic motion. To this end, the method relies on direct numerical time integration and on the Newton-Raphson algorithm.

Starting from some assumed initial conditions $\mathbf{z}_{p 0}^{(0)}$, the motion $\mathbf{z}_{p}^{(0)}\left(t, \mathbf{z}_{p 0}^{(0)}\right)$ at the assumed period $T^{(0)}$ can be obtained by numerical time integration methods (e.g., Runge-Kutta or Newmark schemes). In general, the initial guess $\left(\mathbf{z}_{p 0}^{(0)}, T^{(0)}\right)$ does not satisfy the periodicity condition (5). This is illustrated in Fig. 7 for a Duffing oscillator

$$
\ddot{x}+x+0.5 x^{3}=0
$$




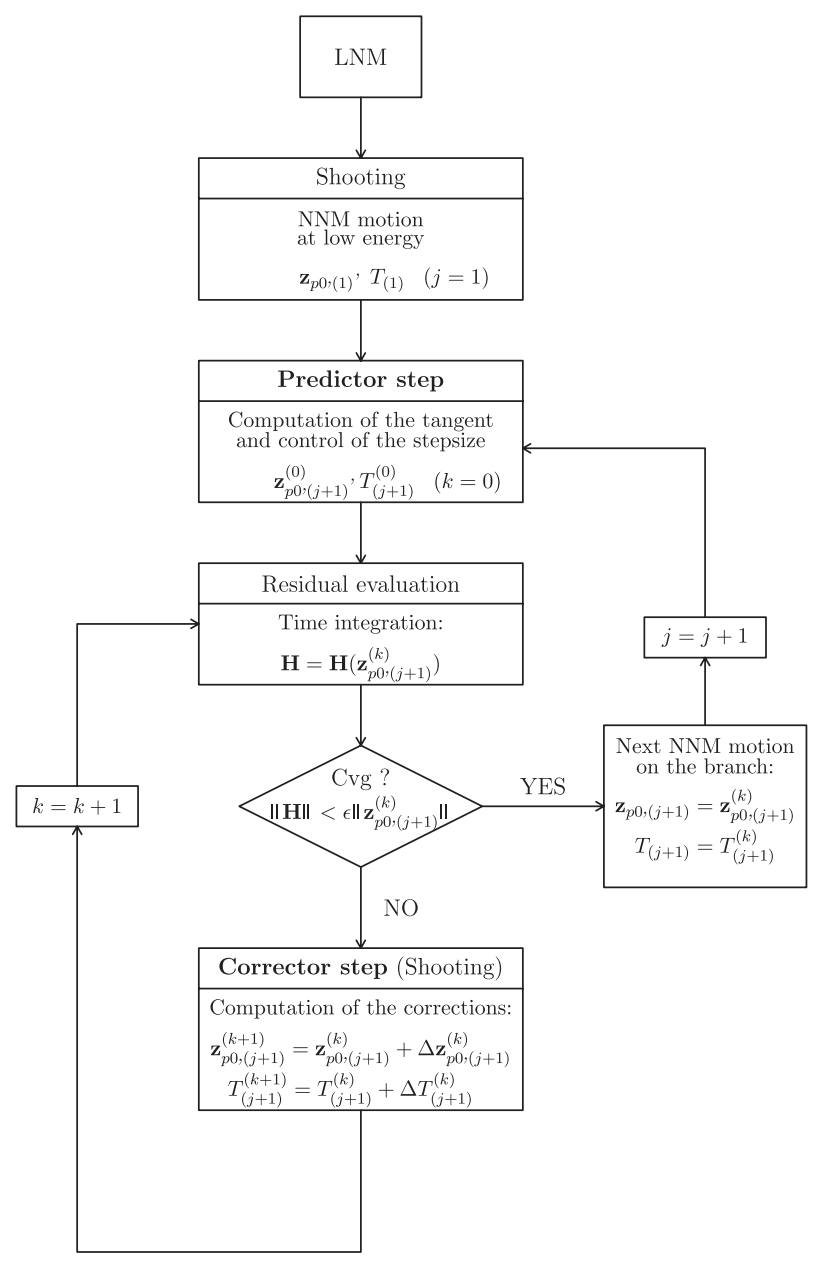

Fig. 6. Algorithm for NNM computation.
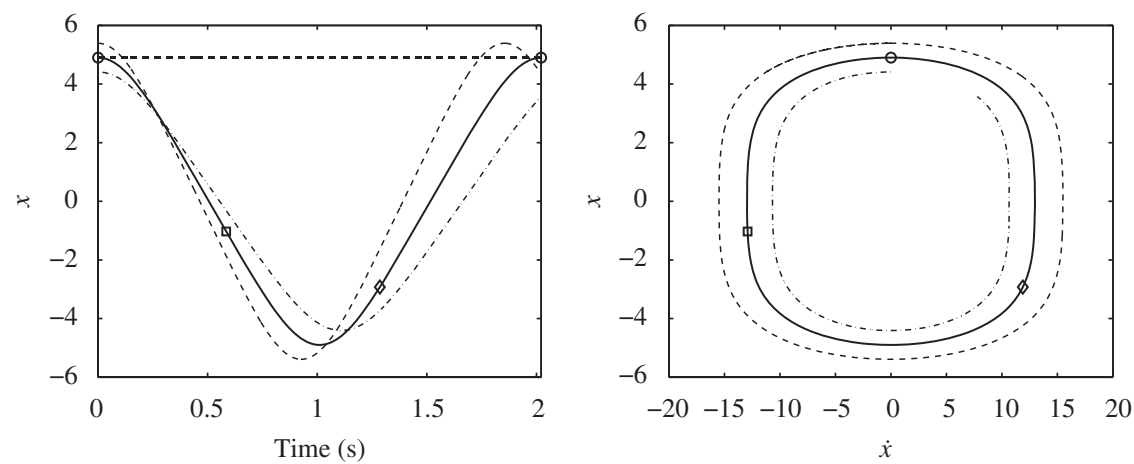

Fig. 7. Solutions of the Duffing oscillator for different initial conditions. Left plot: time series; right plot: phase space. $\longrightarrow$ : periodic solution for $[x(0) \dot{x}(0)]=[4.90090]$ and $T=2.0215 \mathrm{~s} ;-\cdots$ : solution for $[x(0) \dot{x}(0)]=1.1 \times[4.90090] ;-\cdot-\cdot-$ : solution for $[x(0) \dot{x}(0)]=0.9 \times[4.90090]$. Markers represent different initial conditions of the periodic solution; $\circ:\left[\begin{array}{l}x \\ \dot{x}\end{array}\right]=[4.90090] ; \square:[x \dot{x}]=[-1.0313-12.9188] ; \diamond:[x \dot{x}]=[-2.925911 .8894]$.

Two pairs of initial conditions, $[x(0) \dot{x}(0)]=0.9 \times[4.90090]$ and $[x(0) \dot{x}(0)]=1.1 \times[4.90090]$, are two approximations to the actual solution, $[x(0) \dot{x}(0)]=[4.9009$ 0], for the current period $T=2.0215 \mathrm{~s}$. The former (latter) approximation yields a motion with a too large (small) period. 
A Newton-Raphson iteration scheme is therefore to be used to correct an initial guess and to converge to the actual solution. The corrections $\Delta \mathbf{z}_{p 0}^{(0)}$ and $\Delta T^{(0)}$ are found by expanding the nonlinear function

$$
\mathbf{H}\left(\mathbf{z}_{p 0}^{(0)}+\Delta \mathbf{z}_{p 0}^{(0)}, T^{(0)}+\Delta T^{(0)}\right)=0
$$

in Taylor series

$$
\mathbf{H}\left(\mathbf{z}_{p 0}^{(0)}, T^{(0)}\right)+\left.\frac{\partial \mathbf{H}}{\partial \mathbf{z}_{p 0}}\right|_{\left(\mathbf{z}_{p 0}^{(0)}, T^{(0)}\right)} \Delta \mathbf{z}_{p 0}^{(0)}+\left.\frac{\partial \mathbf{H}}{\partial T}\right|_{\left(\mathbf{z}_{p 0}^{(0)}, T^{(0)}\right)} \Delta T^{(0)}+\text { H.O.T. }=0
$$

and neglecting higher-order terms (H.O.T.).

The initial conditions $\mathbf{z}_{p 0}$ and the period $T$ characterizing the periodic solution are computed through the iterative procedure

$$
\mathbf{z}_{p 0}^{(k+1)}=\mathbf{z}_{p 0}^{(k)}+\Delta \mathbf{z}_{p 0}^{(k)} \quad \text { and } \quad T^{(k+1)}=T^{(k)}+\Delta T^{(k)}
$$

with

$$
\left.\frac{\partial \mathbf{H}}{\partial \mathbf{z}_{p 0}}\right|_{\left(\mathbf{z}_{p 0}^{(k)}, T^{(k)}\right)} \Delta \mathbf{z}_{p 0}^{(k)}+\left.\frac{\partial \mathbf{H}}{\partial T}\right|_{\left(\mathbf{z}_{p 0}^{(k)}, T^{(k)}\right)} \Delta T^{(k)}=-\mathbf{H}\left(\mathbf{z}_{p 0}^{(k)}, T^{(k)}\right)
$$

where $k$ is the shooting iteration index. Convergence is achieved when $\mathbf{H}\left(\mathbf{z}_{p 0}, T\right) \approx 0$ to the desired accuracy. In the neighborhood of the solution, the convergence is fast (i.e., quadratic convergence for an exact evaluation of the Jacobian matrix). However, it should be kept in mind that the Newton-Raphson method is a local algorithm; the convergence is guaranteed only when the initial guess is sufficiently close to the solution.

Each shooting iteration involves the time integration of the equations of motion to evaluate the current shooting residue $\mathbf{H}\left(\mathbf{z}_{p 0}^{(k)}, T^{(k)}\right)=\mathbf{z}_{p}^{(k)}\left(T^{(k)}, \mathbf{z}_{p 0}^{(k)}\right)-\mathbf{z}_{p 0}^{(k)}$. As evidenced by Eq. (10), the shooting method also requires the evaluation of the partial derivatives of $\mathbf{H}\left(\mathbf{z}_{0}, T\right)=\mathbf{z}\left(T, \mathbf{z}_{0}\right)-\mathbf{z}_{0}$. The $2 n \times 1$ vector $\partial \mathbf{H} / \partial T$ is given by

$$
\begin{aligned}
\frac{\partial \mathbf{H}}{\partial T}\left(\mathbf{z}_{0}, T\right) & =\left.\frac{\partial \mathbf{z}\left(t, \mathbf{z}_{0}\right)}{\partial t}\right|_{t=T} \\
& =\mathbf{g}\left(\mathbf{z}\left(T, \mathbf{z}_{0}\right)\right)
\end{aligned}
$$

The $2 n \times 2 n$ Jacobian matrix $\partial \mathbf{H} / \partial \mathbf{z}_{0}$ is provided by

$$
\frac{\partial \mathbf{H}}{\partial \mathbf{z}_{0}}\left(\mathbf{z}_{0}, T\right)=\left.\frac{\partial \mathbf{z}\left(t, \mathbf{z}_{0}\right)}{\partial \mathbf{z}_{0}}\right|_{t=T}-\mathbf{I}
$$

where $\mathbf{I}$ is the $2 n \times 2 n$ identity matrix. There are basically two means of computing the Jacobian matrix $\partial \mathbf{z}\left(t, \mathbf{z}_{0}\right) / \partial \mathbf{z}_{0}$.

1. This matrix represents the variation of the solution $\mathbf{z}\left(t, \mathbf{z}_{0}\right)$ at time $t$ when the initial conditions $\mathbf{z}_{0}$ are perturbed. It can, therefore, be evaluated through a numerical finite-difference analysis by perturbing successively each of the initial conditions and integrating the equations of motion [5].

2. An alternative computation is obtained by differentiating the equations of motion (3) with respect to the initial conditions $\mathbf{z}_{0}$

$$
\frac{\partial}{\partial \mathbf{z}_{0}}\left[\dot{\mathbf{z}}\left(t, \mathbf{z}_{0}\right)\right]=\frac{\partial}{\partial \mathbf{z}_{0}}\left[\mathbf{g}\left(\mathbf{z}\left(t, \mathbf{z}_{0}\right)\right)\right]
$$

It follows

$$
\frac{d}{d t}\left[\frac{\partial \mathbf{z}\left(t, \mathbf{z}_{0}\right)}{\partial \mathbf{z}_{0}}\right]=\left.\frac{\partial \mathbf{g}(\mathbf{z})}{\partial \mathbf{z}}\right|_{\mathbf{z}\left(t, \mathbf{z}_{0}\right)}\left[\frac{\partial \mathbf{z}\left(t, \mathbf{z}_{0}\right)}{\partial \mathbf{z}_{0}}\right]
$$

with

$$
\frac{\partial \mathbf{z}\left(0, \mathbf{z}_{0}\right)}{\partial \mathbf{z}_{0}}=\mathbf{I}
$$

since $\mathbf{z}\left(0, \mathbf{z}_{0}\right)=\mathbf{z}_{0}$. Hence, the matrix $\partial \mathbf{z}\left(t, \mathbf{z}_{0}\right) / \partial \mathbf{z}_{0}$ at $t=T$ can be obtained by numerically integrating over $T$ the initialvalue problem defined by the ordinary differential equations (ODEs) (14) with the initial conditions (15).

In addition to the integration of the current solution $\mathbf{z}\left(t, \mathbf{x}_{0}\right)$ of (3), these two methods for computing $\partial \mathbf{z}\left(t, \mathbf{z}_{0}\right) / \partial \mathbf{z}_{0}$ require $2 n$ numerical integrations of $2 n$-dimensional dynamical systems, which may be computationally intensive for large systems. However, equations (14) are linear ODEs, and their numerical integration is thus less expensive. The numerical cost can be further reduced if the solution of Eq. (14) is computed together with the solution of the nonlinear equations of motion in a single simulation [31]. We note that the finite-difference procedure is required when $\mathbf{g}$ is nondifferentiable, i.e., when the nonlinearities are nonsmooth [5,32]. 
In the present case, the phase of the periodic solutions is not fixed. If $\mathbf{z}(t)$ is a solution of the autonomous system (3), then $\mathbf{z}(t+\Delta t)$ is geometrically the same solution in phase space for any $\Delta t$. The initial conditions $\mathbf{z}_{p 0}$ can be arbitrarily chosen anywhere on the periodic solution. This is illustrated in Fig. 7 for the Duffing oscillator (6) where different initial conditions corresponding to the same periodic solution are shown. Hence, an additional condition has to be specified in order to remove the arbitrariness of the initial conditions. Mathematically, the system (10) of $2 n$ equations with $2 n+1$ unknowns needs a supplementary equation, termed the phase condition.

Different phase conditions have been proposed in the literature [4,5]. For instance, the simplest one consists in setting one component of the initial conditions vector to zero, as in [17]. This is illustrated in Fig. 7 where the depicted periodic solution of the Duffing oscillator is characterized by a zero initial velocity. A phase condition particularly suitable for the NNM computation is utilized in the present study and is discussed in Section 3.3.2.

In summary, the NNM computation is carried out by solving the augmented two-point boundary-value problem defined by

$$
\mathbf{F}\left(\mathbf{z}_{p 0}, T\right) \equiv\left\{\begin{array}{l}
\mathbf{H}\left(\mathbf{z}_{p 0}, T\right)=0 \\
h\left(\mathbf{z}_{p 0}\right)=0
\end{array}\right.
$$

where $h\left(\mathbf{z}_{p 0}\right)=0$ is the phase condition.

An important characteristic of NNMs is that they can be stable or unstable, which is in contrast to linear theory where all modes are neutrally stable. In this context, instability means that small perturbations of the initial conditions that generate the NNM motion lead to the elimination of the mode oscillation. Nonetheless, the unstable NNMs can be computed using the shooting procedure.

The stability analysis can be performed when an NNM motion has been computed by the shooting algorithm. The monodromy matrix $\Phi_{T}$ of a periodic orbit $\mathbf{z}_{p}\left(t, \mathbf{z}_{p 0}\right)$ of period $T$ is defined by its $2 n \times 2 n$ Jacobian matrix evaluated at $t=T$

$$
\Phi_{T}\left(\mathbf{z}_{p 0}\right)=\left.\frac{\partial \mathbf{z}_{p}\left(t, \mathbf{z}_{p 0}\right)}{\partial \mathbf{z}_{p 0}}\right|_{t=T}
$$

Perturbing the initial conditions with the vector $\Delta \mathbf{z}_{0}$ and expanding the perturbed solution $\mathbf{z}\left(T, \mathbf{z}_{p 0}+\Delta \mathbf{z}_{0}\right)$ in Taylor series yields

$$
\Delta \mathbf{z}(T)=\Phi_{T}\left(\mathbf{z}_{p 0}\right) \Delta \mathbf{z}_{0}+\mathscr{O}\left(\left\|\Delta \mathbf{z}_{0}\right\|^{2}\right)
$$

where $\Delta \mathbf{z}(T)=\mathbf{z}\left(T, \mathbf{z}_{p 0}+\Delta \mathbf{z}_{0}\right)-\mathbf{z}_{p}\left(T, \mathbf{z}_{p 0}\right)$.

Eq. (18) shows that the monodromy matrix provides the first-order variation of the periodic solution after one period. After $m$ periods, one obtains

$$
\Delta \mathbf{z}(m T)=\left[\Phi_{T}\left(\mathbf{z}_{p 0}\right)\right]^{m} \Delta \mathbf{z}_{0}+\mathcal{O}\left(\left\|\Delta \mathbf{z}_{0}\right\|^{2}\right)
$$

The linear stability of the periodic solution calculated by the shooting algorithm is studied by computing the eigenvalues of its monodromy matrix $\Phi_{T}$. The $2 n$ eigenvalues, termed Floquet multipliers, provide the exponential variations of the perturbations along the eigendirections of the monodromy matrix. If a Floquet multiplier has a magnitude larger than one, then the periodic solution is unstable; otherwise, it is stable in the linear sense.

\subsection{Continuation of periodic solutions}

The conservative system (3) comprises at least $n$ different families of periodic orbits (i.e., NNMs), which can be regarded as nonlinear extensions of the LNMs of the underlying linear system. Due to the frequency-energy dependence, the modal parameters of an NNM vary with the total energy. An NNM family, governed by Eq. (16), therefore traces a curve, termed an NNM branch, in the $(2 n+1)$-dimensional space of initial conditions and period $\left(\mathbf{z}_{p 0}, T\right)$. As stated before, there may also exist additional NNMs (i.e., bifurcating NNMs) that are essentially nonlinear with no linear counterparts.

In this study, the NNMs are determined using methods for the numerical continuation of periodic motions (also called path-following methods) [4,5,33]. Starting from the corresponding LNM at low energy, the computation is carried out by finding successive points $\left(\mathbf{z}_{p 0}, T\right)$ of the NNM branch. The space $\left(\mathbf{z}_{p 0}, T\right)$ is termed the continuation space.

Different methods for numerical continuation have been proposed in the literature. The so-called pseudo-arclength continuation method is used herein.

\subsubsection{Sequential continuation}

The simplest and most intuitive continuation technique is the sequential continuation method. This procedure is first explained due to its straightforward implementation. Moreover, it provides the fundamental concepts of continuation methods.

The sequential continuation of the periodic solutions governed by Eq. (16) is carried out in three steps:

1. A periodic solution $\left(\mathbf{z}_{p 0,(1)}, T_{(1)}\right)$ at sufficiently low energy (i.e., in the neighborhood of one LNM) is first computed using the shooting method. The period and initial conditions of the selected LNM are chosen as an initial guess. 
2. The period is incremented, $T_{(j+1)}=T_{(j)}+\Delta T$.

3. From the current solution $\left(\mathbf{z}_{p 0,(j)}, T_{(j)}\right)$, the next solution $\left(\mathbf{z}_{p 0,(j+1)}, T_{(j+1)}\right)$ is determined by solving Eq. (16) using the shooting method with the period fixed:

$$
\mathbf{z}_{p 0,(j+1)}^{(k+1)}=\mathbf{z}_{p 0,(j+1)}^{(k)}+\Delta \mathbf{z}_{p 0,(j+1)}^{(k)}
$$

where

$$
\left.\frac{\partial \mathbf{F}}{\partial \mathbf{z}_{p 0}}\right|_{\left(\mathbf{z}_{p 0,(j+1)}^{(k)}, T_{(j+1)}\right)} \Delta \mathbf{z}_{p 0,(j+1)}^{(k)}=-\mathbf{F}\left(\mathbf{z}_{p 0,(j+1)}^{(k)}, T_{(j+1)}\right)
$$

The initial conditions of the previous periodic solution are used as a prediction $\mathbf{z}_{p 0,(j+1)}^{(0)}=\mathbf{z}_{p 0,(j)}$. Superscript $k$ is the iteration index of the shooting procedure, whereas subscript $j$ is the index along the NNM branch.

Eventually, one NNM branch is computed.

\subsubsection{Pseudo-arclength continuation}

The sequential continuation method parameterizes an NNM branch using the period $T$. It has two main drawbacks:

1. Because the convergence of the Newton-Raphson procedure depends critically on the closeness of the initial guess to the actual solution, the sequential continuation requires fairly small increments $\Delta T$.

2. Because the value of the period is fixed during the Newton-Raphson corrections, it is unable as such to deal with turning points. This is illustrated in Fig. 8 where no solution exists for a period larger than the period at the turning point.

For better performance, a continuation algorithm uses a better prediction than the last computed solution. In addition, corrections of the period are also considered during the shooting process. The pseudo-arclength continuation method relies on these two improvements in order to optimize the path following of the branch.

Starting from a known solution $\left(\mathbf{z}_{p 0,(j)}, T_{(j)}\right)$, the next periodic solution $\left(\mathbf{z}_{p 0,(j+1)}, T_{(j+1)}\right)$ on the branch is computed using a predictor step and a corrector step.

Predictor step: At step $j$, a prediction $\left(\tilde{\mathbf{z}}_{p 0,(j+1)}, \tilde{T}_{(j+1)}\right)$ of the next solution $\left(\mathbf{z}_{p 0,(j+1)}, T_{(j+1)}\right)$ is generated along the tangent vector to the branch at the current point $\mathbf{z}_{p 0,(j)}$

$$
\left[\begin{array}{c}
\tilde{\mathbf{z}}_{p 0,(j+1)} \\
\tilde{T}_{(j+1)}
\end{array}\right]=\left[\begin{array}{c}
\mathbf{z}_{p 0,(j)} \\
T_{(j)}
\end{array}\right]+s_{(j)}\left[\begin{array}{c}
\mathbf{p}_{z,(j)} \\
p_{T,(j)}
\end{array}\right]
$$

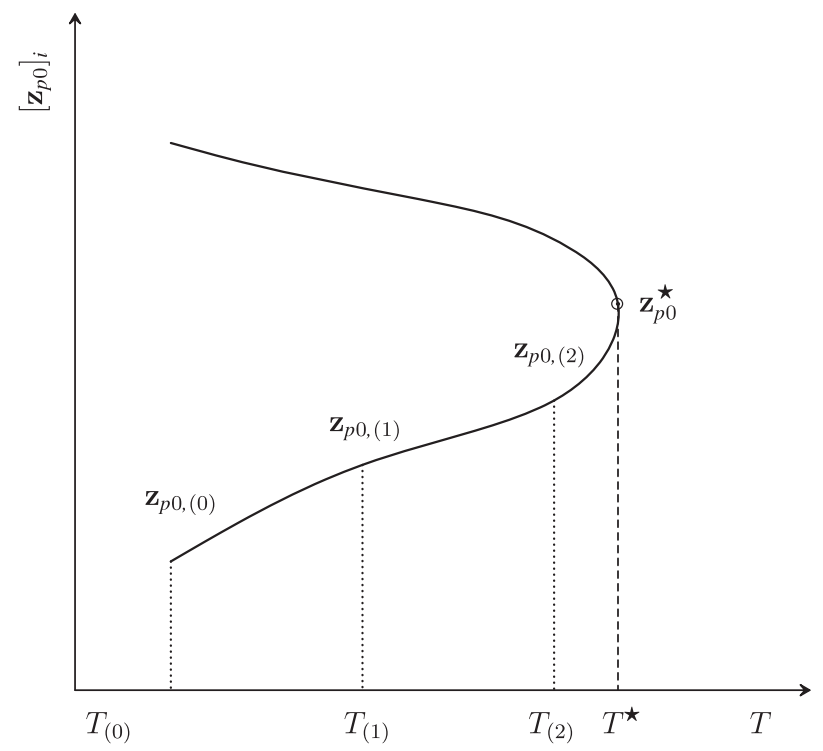

Fig. 8. Turning point $\left(T^{*}, \mathbf{z}_{p 0}^{\star}\right)$ in the continuation space. Failure of the sequential continuation for $T \geqslant T^{\star}$. 
where $s_{(j)}$ is the predictor stepsize. The tangent vector $\mathbf{p}_{(j)}=\left[\mathbf{p}_{z,(j)}^{*} p_{T,(j)}\right]^{*}$ to the branch defined by (16) is solution of the system

$$
\left[\begin{array}{cc}
\left.\frac{\partial \mathbf{H}}{\partial \mathbf{z}_{p 0}}\right|_{\left(\mathbf{z}_{p 0,(j)}, T_{(j)}\right)} & \left.\frac{\partial \mathbf{H}}{\partial T}\right|_{\left(\mathbf{z}_{p 0,(j)}, T_{(j)}\right)} \\
\left.\frac{\partial h^{*}}{\partial \mathbf{z}_{p 0}}\right|_{\left(\mathbf{z}_{p 0,(j)}\right)} & 0
\end{array}\right]\left[\begin{array}{l}
\mathbf{p}_{z,(j)} \\
p_{T,(j)}
\end{array}\right]=\left[\begin{array}{l}
\mathbf{0} \\
0
\end{array}\right]
$$

with the condition $\left\|\mathbf{p}_{(j)}\right\|=1$. The star denotes the transpose operator. This normalization can be taken into account by fixing one component of the tangent vector and solving the resulting overdetermined system using the Moore-Penrose matrix inverse; the tangent vector is then normalized to 1. For illustration, the predictor step is shown schematically in Fig. 9.

Corrector step: The prediction is corrected by a shooting procedure in order to solve Eq. (16) in which the variations of the initial conditions, and the period are forced to be orthogonal to the predictor step. At iteration $k$, the corrections

$$
\begin{aligned}
& \mathbf{z}_{p 0,(j+1)}^{(k+1)}=\mathbf{z}_{p 0,(j+1)}^{(k)}+\Delta \mathbf{z}_{p 0,(j+1)}^{(k)} \\
& T_{(j+1)}^{(k+1)}=T_{(j+1)}^{(k)}+\Delta T_{(j+1)}^{(k)}
\end{aligned}
$$

are computed by solving the overdetermined linear system using the Moore-Penrose matrix inverse

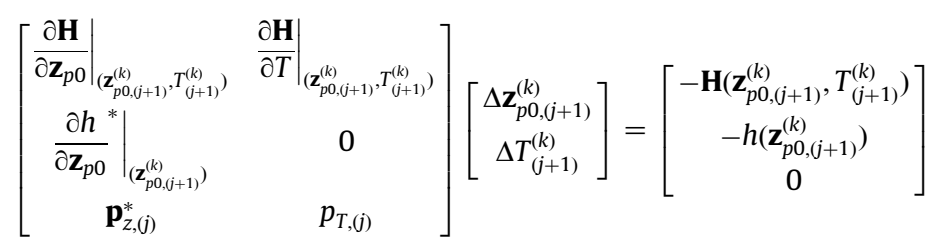

where the prediction is used as initial guess, i.e, $\mathbf{z}_{p 0,(j+1)}^{(0)}=\tilde{\mathbf{z}}_{p 0,(j+1)}$ and $T_{(j+1)}^{(0)}=\tilde{T}_{(j+1)}$.

The last equation in (25) corresponds to the orthogonality condition for the corrector step. We note that the partial derivatives in Eq. (25) are evaluated numerically, as explained previously.

This iterative process is carried out until convergence is achieved. The convergence test is based on the relative error of the periodicity condition:

$$
\frac{\left\|\mathbf{H}\left(\mathbf{z}_{p 0}, T\right)\right\|}{\left\|\mathbf{z}_{p 0}\right\|}=\frac{\left\|\mathbf{z}_{p}\left(T, \mathbf{z}_{p 0}\right)-\mathbf{z}_{p 0}\right\|}{\left\|\mathbf{z}_{p 0}\right\|}<\varepsilon
$$

where $\varepsilon$ is the prescribed relative precision.

For illustration, the corrector step is shown schematically in Fig. 9.

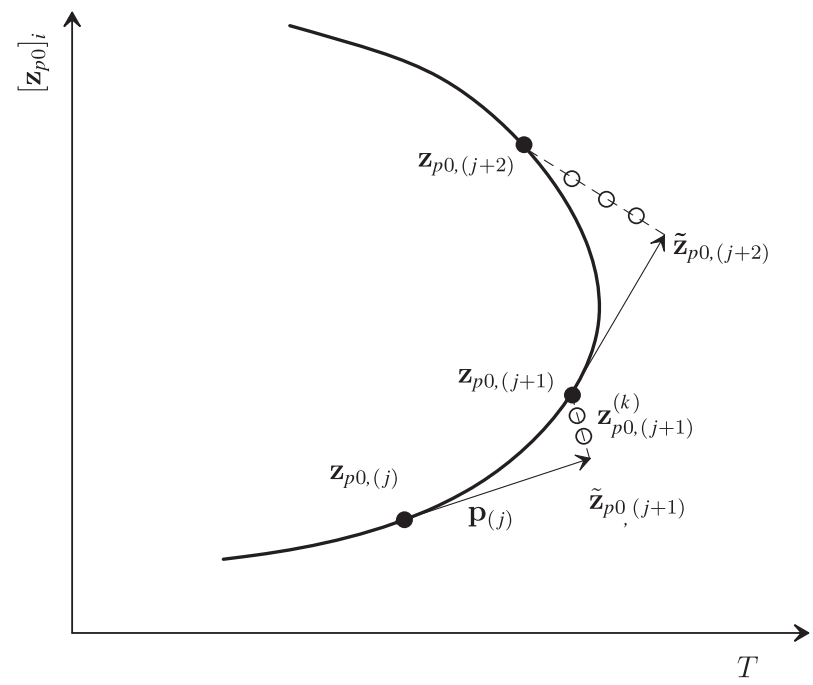

Fig. 9. Pseudo-arclength continuation method: branch $(-)$ with a turning point; predictor step $(\rightarrow)$ tangent to the branch; corrector steps $(\circ \circ \circ)$ perpendicular to the predictor step. 


\subsection{An integrated approach for the NNM computation}

\subsubsection{Basic algorithm}

The algorithm proposed for the computation of NNM motions is a combination of shooting and pseudo-arclength continuation methods, as shown in Fig. 6. Starting from the LNM motion at low energy, there are two steps within the algorithm:

1. The predictor step is global and goes from one NNM motion at a specific energy level to another NNM motion at a somewhat different energy level. For an efficient and robust NNM continuation, the stepsize $s_{(j)}$ is to be carefully controlled. A small stepsize leads to a small number of corrector iterations, but it requires a large number of continuation steps to follow an NNM branch. For a large stepsize, the number of corrector iterations is high, and the convergence is slow. The Newton-Raphson procedure may even break down if the prediction is not close enough to the actual solution. Continuation may, therefore, be computationally intensive in both cases. The stepsize has to be adjusted, possibly in an automatic and flexible manner. Various adaptive stepsize control procedures are discussed in $[4,33]$.

2. The corrector step is local and refines the prediction to obtain the actual solution at a specific energy level. The size of the corrections during the corrector step is determined by the solutions of the overdetermined system (25).

This algorithm is applied to the Duffing oscillator (6) in Fig. 10. The phase condition used in this example consists in enforcing the initial velocity $\dot{x}_{p 0}$ to be zero. The continuation space is, therefore, composed of the initial displacement $x_{p 0}$ and the period $T$. The initial guess used to start the procedure is obtained at low energy where the system responds practically as the underlying linear system, which has an eigenfrequency of $1 \mathrm{rad} / \mathrm{s}$ (i.e., a period of $T=6.28 \mathrm{~s}$ ).

NNM representation: So far, the NNMs have been considered as branches in the continuation space $\left(\mathbf{z}_{p 0}, T\right)$. As explained in Section 2.2.1, an appropriate graphical depiction of the NNMs is to represent them in a FEP. This FEP can be computed in a straightforward manner: (i) the conserved total energy is computed from the initial conditions realizing the NNM motion and (ii) the frequency of the NNM motion is calculated directly from the period.

Numerical time integration: A widely used method for solving first-order equations such as Eq. (3) is the Runge-Kutta scheme. In structural dynamics where second-order systems are encountered, Newmark's family of methods is probably the most widespread technique for solving linear and nonlinear large-scale stiff structural systems [34]. This family of numerical time integration methods is considered in this study.

The precision of the integration scheme, which is chosen by the end-user, directly influences the accuracy of the NNM computation. In fact, the computed solution can be regarded as an exact solution if the sampling frequency used to integrate the equations is sufficiently high. This is practically the only approximation in the proposed algorithm.

Step control: Unlike sequential continuation, the evolution path of this predictor-corrector method is parameterized by the distance $s_{(j)}$ along the tangent predictor, also referred to as arclength continuation parameter in the literature. As mentioned previously, the stepsize has to be carefully controlled for a robust and efficient NNM computation.

The stepsize control used herein relies on the evaluation of the convergence quality by the number of iterations of the corrector step. The stepsize is controlled so that the corrector step requires on average the desirable number of iterations $N^{\star}$. At each step, the stepsize is updated according to the ratio between the desirable number $N^{*}$ and the previous number $N_{(j-1)}$ of iterations:

$$
s_{(j)}=\left(\frac{N^{*}}{N_{(j-1)}}\right) s_{(j-1)}
$$
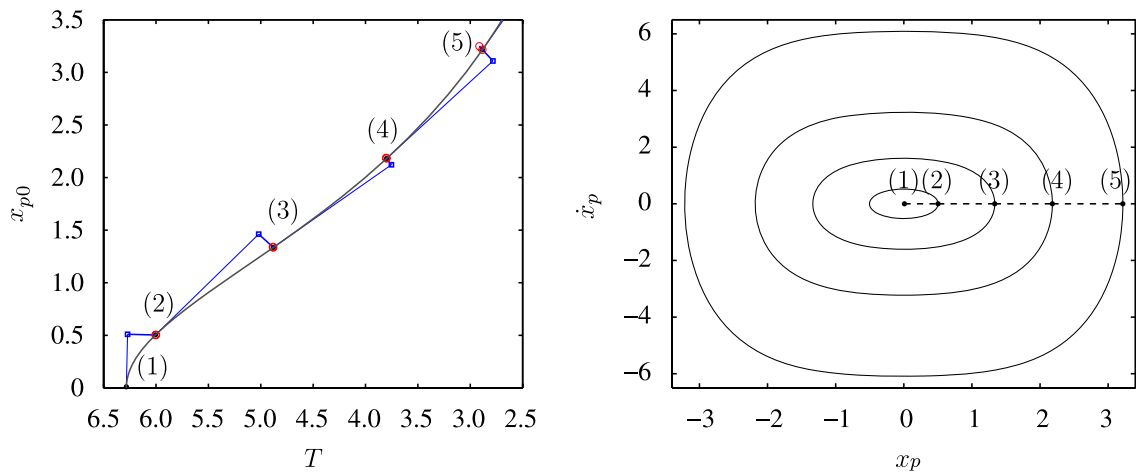

Fig. 10. Continuation of periodic solutions for the Duffing oscillator (phase condition: the initial velocity $\dot{x}_{p 0}=0$; initial guess point: $\left[x_{p 0} \dot{x}_{p 0}\right]=[0.010]$ and $T=2 \pi \mathrm{s}$ ). Left plot: initial displacement $x_{p 0}$ as function of the period of motion (continuation space); exact branch $(-)$ and computed points (•); predictor steps $(\square)$ and corrector steps ( $($ ). Right plot: family of periodic motions in the phase space; corresponding path following (- - -) and related periodic solutions (-). 
In practice, the ratio $r=N^{*} / N_{(j-1)}$ is often bounded to make the adaptation stepsize more robust and to prevent the continuation from jumping between different branches. ${ }^{1}$ The stepsize can also be bounded $\left(s_{(j)}<s_{\max }\right)$ to obtain enough discretized points on the branch during the continuation. In case of no convergence (i.e., when the residue increases or when the process requires more correction iterations than the prescribed maximum $N_{\max }$ ), the stepsize is halved until convergence is achieved.

As a final remark, we note that the sign of the stepsize is chosen in order to follow the branch in the same direction, i.e.,

$$
\left[s_{(j)} \mathbf{p}_{(j)}\right]^{*}\left[s_{(j-1)} \mathbf{p}_{(j-1)}\right]>0
$$

According to the previous predictor step and the current tangent vector, the sign of $s_{(j)}$ is therefore given by

$$
\operatorname{sign}\left(s_{(j)}\right)=\operatorname{sign}\left(s_{(j-1)} \mathbf{p}_{(j)}^{*} \mathbf{p}_{(j-1)}\right)
$$

\subsubsection{Reduction of the computational burden}

As discussed in Section 2, a seemingly simple system such as system (2) can exhibit complicated NNM motions. This 2DOF system possesses branches of fundamental NNM motions, termed backbone branches, which are the nonlinear extension of the LNM motions. These branches coexist with a countable infinity of branches of internally resonant NNMs, termed tongues, with no counterpart in linear theory. In view of the infinite number of tongues and their sensitivity to small perturbations with increasing orders of internal resonance, an extensive computation of the tongues is certainly debatable. Although interesting, this discussion is beyond the scope of this paper.

The algorithm described so far may become computationally intensive when dealing with large-scale systems (i.e., systems with many DOFs). As a practical and computationally tractable calculation of the NNM motions is targeted, two properties of some NNM families can be exploited to speed up the computation:

1. All symmetric NNM branches Snm (see, e.g., Fig. 4 and the top plot in Fig. 5) obey the symmetry condition

$$
\mathbf{z}_{p}\left(\frac{T}{2}, \mathbf{z}_{p 0}\right)+\mathbf{z}_{p 0}=0
$$

For these branches, the shooting procedure can be performed over the half-period $T / 2$ by searching the initial conditions and the period $T$ that solve this modified periodicity condition. As the time integrations represent the main computational cost of the algorithm, this modified periodicity condition reduces the computational burden by a factor close to 2 .

2. For branches of NNMs represented by an open loop in the configuration space (see, e.g., the first two NNM motions in Fig. 5), a suitable phase condition is to set all the velocities to zero. The initial velocities are eliminated from the unknowns of the linear systems to solve at each Newton-Raphson iteration. These systems have, therefore, $2 n+1$ equations with $n+1$ unknowns $\mathbf{x}_{p 0}$ and $T$.

One advantage is that these modifications can be very naturally integrated in the basic algorithm in Fig. 6 .

In summary, two variants of the basic algorithm described in the previous section have been developed:

1. The general strategy consists in (i) carrying out the shooting over the period $T$ and (ii) setting only one velocity to zero. This strategy can compute all possible NNM motions.

2. The practical strategy exploits the modified periodicity and phase conditions. The fundamental NNM motions of nonlinear structures often obey these conditions (e.g., the fundamental NNMs of nonlinear systems with odd nonlinearities are necessarily symmetric, because the loss of symmetry requires the so-called symmetry-breaking bifurcation).

Targeting a reduction of the computational burden, but without lack of generality, an approach that integrates the two variants is used. Starting from the LNMs, the fundamental NNM motions are first computed using the practical strategy; a subset of tongues can also be computed using this methodology. We note that the application of this strategy should often suffice for most engineering structures.

When a detailed analysis of the unsymmetric NNMs and those represented by a closed curve in the configuration space is required, the general strategy can then be utilized for computing these NNMs.

\section{Numerical experimentations}

In what follows, the proposed NNM computation method is demonstrated using four different nonlinear vibrating systems, namely a weakly nonlinear 2DOF system, a 2DOF system with an essential nonlinearity, a discrete model of a nonlinear bladed disk and a nonlinear cantilever beam discretized by the finite element method.

\footnotetext{
${ }^{1}$ Another possibility to avoid branch jumping phenomena is to limit the angle between consecutive predictor steps.
} 


\subsection{Weakly nonlinear 2DOF system}

The system is illustrated in Fig. 1. The governing equations of motion are

$$
\begin{aligned}
& \ddot{x}_{1}+\left(2 x_{1}-x_{2}\right)+0.5 x_{1}^{3}=0 \\
& \ddot{x}_{2}+\left(2 x_{2}-x_{1}\right)=0
\end{aligned}
$$

The two LNMs of the underlying linear system are in-phase and out-of-phase modes for which the two DOFs vibrate with the same amplitude. The natural eigenfrequencies are $f_{1}=1 / 2 \pi \simeq 0.159 \mathrm{~Hz}$ and $f_{2}=\sqrt{3} / 2 \pi \simeq 0.276 \mathrm{~Hz}$.

The integrated approach described in Section 3.3 is applied to this system. The computation of the fundamental NNMs is first performed using the modified phase and periodicity conditions. The in-phase backbone $S 11+$ is depicted in Fig. 11(a), whereas the out-of-phase backbone $S 11$ - is given in Fig. 12. Though a large energy range is investigated, these figures show that the continuation method discretizes the two branches using very few points. Large stepsizes are, therefore, employed, and only a few seconds are required to computed each branch for 100 integration time steps per half-period using a $2 \mathrm{GHz}$ processor. This is an important feature when targeting a computationally tractable calculation of the NNMs. The two backbones are depicted together in the FEP in Fig. 2. The family of in-phase NNM motions is also represented in a threedimensional projection of the phase space in Fig. 11(b) and in the configuration space in Fig. 11(c).

The NNM continuation can now be carried out at higher energy levels. The FEP for the in-phase mode is depicted in Fig. 13. It can be observed that a recurrent series of tongues of internally resonant NNMs (i.e., S31, S51, S71, etc.) continue the backbone branch $S 11+$ through turning points (fold bifurcations). Due to these turning points, smaller stepsizes are necessary, which renders the tongue calculation more demanding computationally. By contrast, at higher energy on $S 11-$, the 1:1 out-of-phase motion persists, and $S 11$ - extends to infinity. The complete FEP calculated using the practical strategy is shown in Fig. 14.

We now move to the general strategy for the computation of unsymmetric NNMs and NNMs represented by a closed curve in the configuration space. These NNMs are generally generated through bifurcations (e.g., symmetry-breaking bifurcations for unsymmetrical NNMs). As the tangent is not uniquely defined at the bifurcation point, they require

a

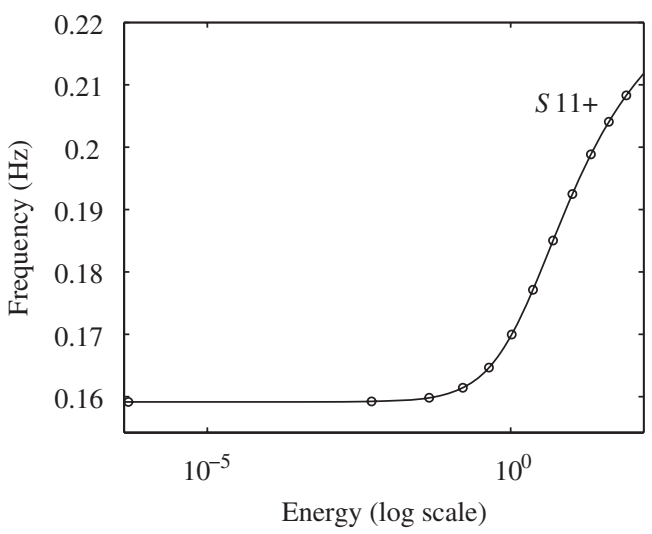

b

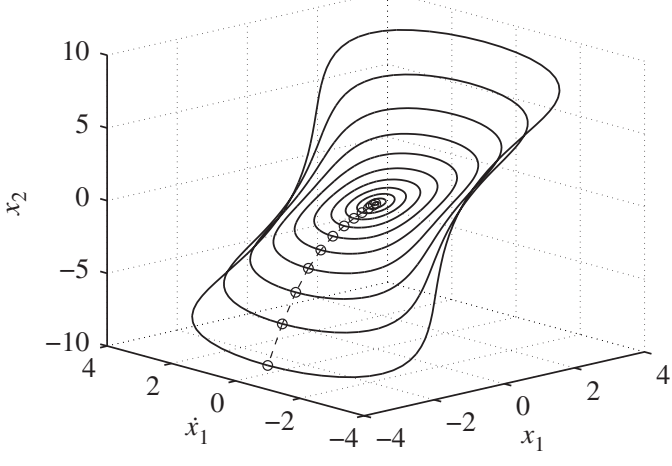

C

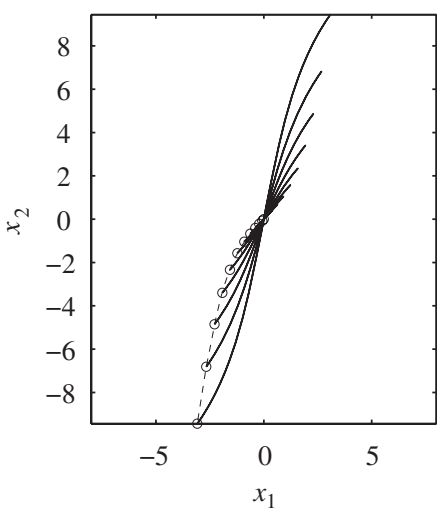

Fig. 11. In-phase NNM motions on $S 11+$ for the 2 DOF system (31). (a) Frequency-energy plot; the computed points with $N^{\star}=4$ are represented by $\circ$. (b) NNM periodic motions represented in a three-dimensional projection of the phase space. (c) NNM modal curves in the configuration space. 


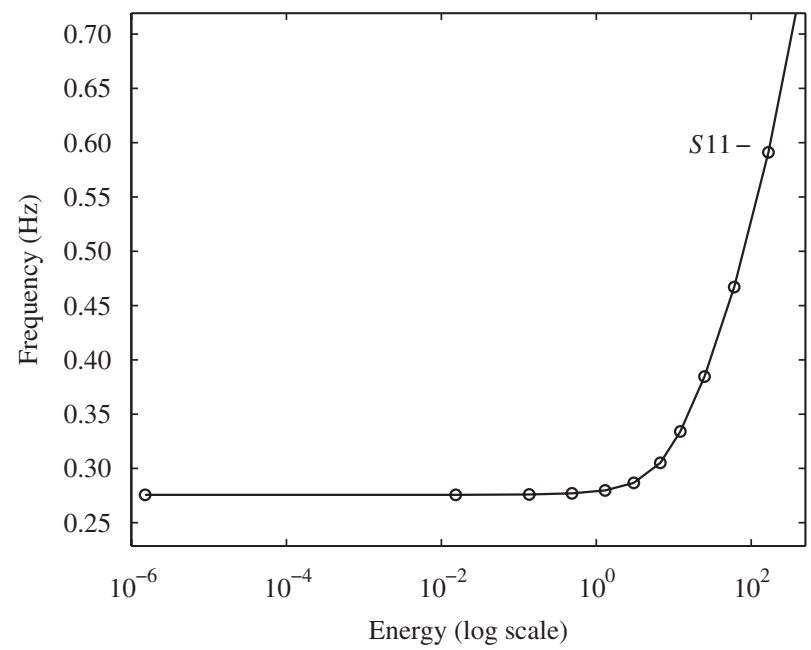

Fig. 12. Frequency-energy plot gathering out-of-phase NNM motions on $S 11-$ for the $2 \mathrm{DOF}$ system (31). The computed points with $N^{\star}=4$ are represented by $\circ$.

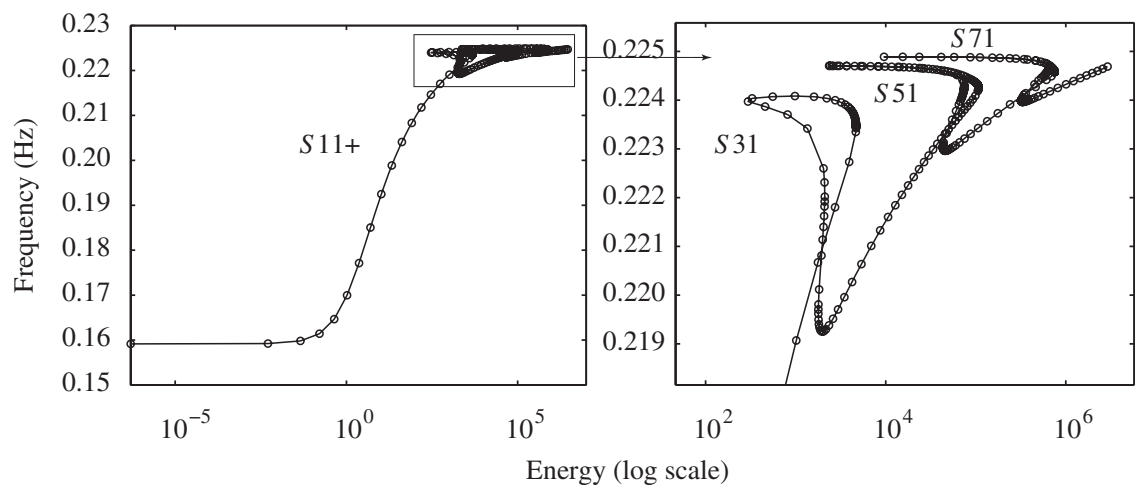

Fig. 13. NNMs at high energy for the 2DOF system (31). Left plot: in-phase backbone S11+ and tongues branches (internally resonant NNMs). Right plot: close-up of the recurrent series of tongues (S31, S51 and S71) at high energy. The computed points are represented by $\circ$.

a branching strategy to be effectively computed [4]. In this study, a perturbation technique is used to carry out branch switching, once the bifurcation point is located using the Floquet multipliers. The resulting FEP is displayed in Fig. 3 and shows two unsymmetrical tongues (U21 and U41).

The NNM stability is also briefly discussed. As the monodromy matrix is computed during the continuation procedure, its eigenvalues, the Floquet multipliers, are obtained as a by-product. The stability of the fundamental in-phase and internally resonant NNMs is presented in Fig. 15. Clearly, the bifurcation points, which include fold and symmetry-breaking bifurcations, are characterized by a change of stability. The evolution of the Floquet multipliers along $S 31$ is shown in the complex plane in Fig. 16. This figure shows the mechanism of loss of stability; a pair of Floquet multipliers leaves the unit circle through 1 .

\subsection{Strongly nonlinear 2DOF system}

The method can also deal with strongly nonlinear systems. To this end, a 2DOF system with an essential nonlinearity is considered. The system is depicted in Fig. 17, and its motion is governed by the equations

$$
\begin{aligned}
& m_{1} \ddot{x_{1}}+k_{1} x_{1}+k_{n l_{1}} x_{1}^{3}+k_{n l_{2}}\left(x_{1}-x_{2}\right)^{3}=0 \\
& m_{2} \ddot{x_{2}}+k_{n l_{2}}\left(x_{2}-x_{1}\right)^{3}=0
\end{aligned}
$$

with $m_{1}=k_{1}=k_{n l_{2}}=1, k_{n l_{1}}=0.5$ and $m_{2}=0.05$

The dynamics of such systems have been extensively studied by Vakakis and co-workers [15,26,35,36] using the NNM concept. The motivation for using an essential nonlinearity is that the nonlinear attachment possesses no preferential 


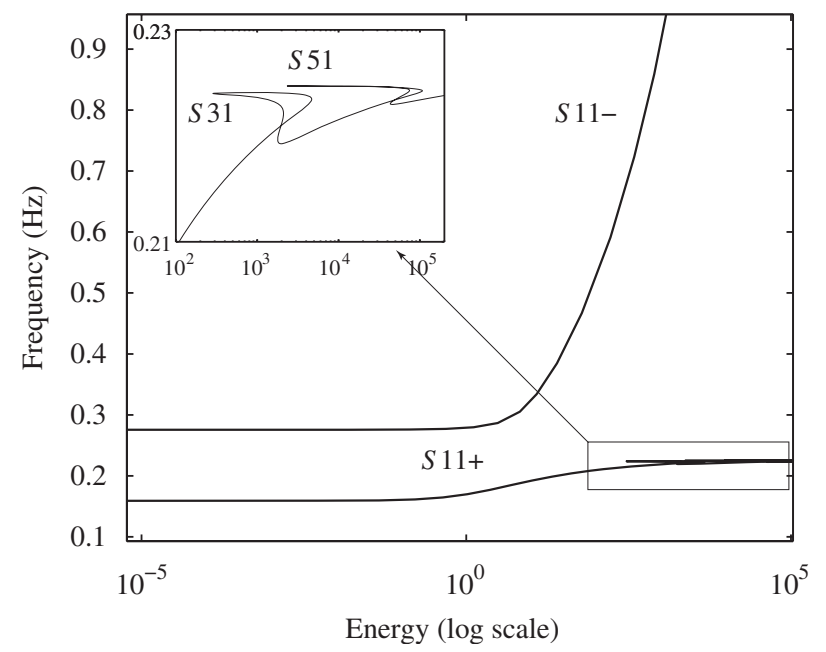

Fig. 14. Frequency-energy plot of the 2DOF system (31).

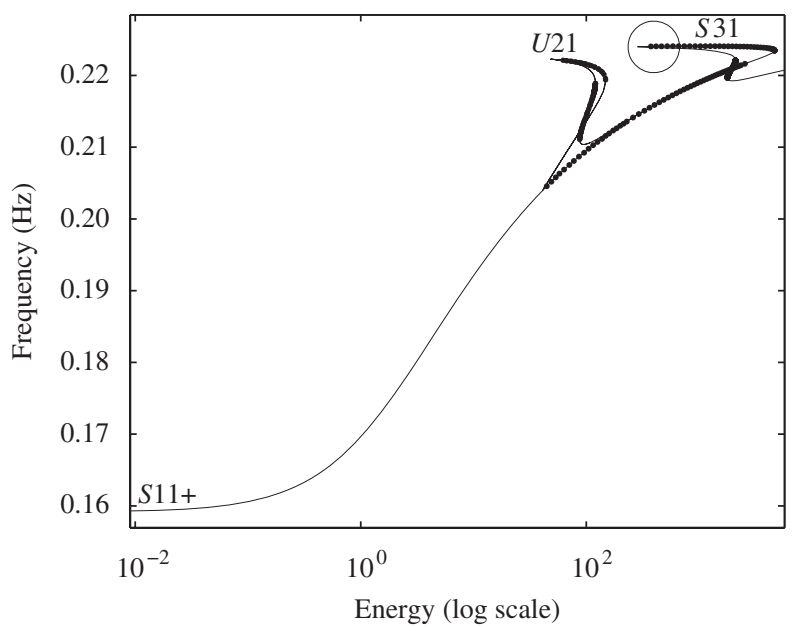

Fig. 15. Close-up of $S 11+$ for the 2 DOF system (31) with stability results ( $\_$: stable NNM motions; • • • unstable NNM motions).

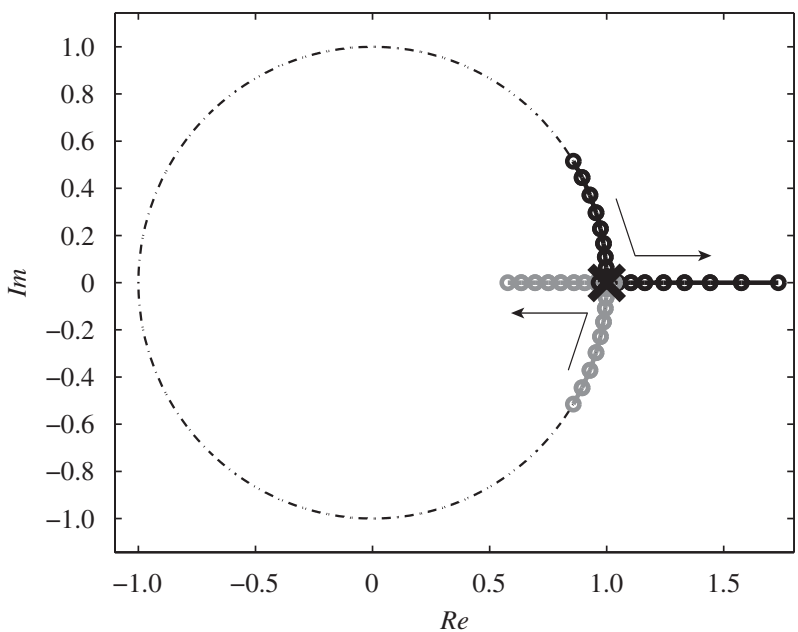

Fig. 16. Evolution of the Floquet multipliers in the complex plane corresponding to the circled area in Fig. 15. One Floquet multiplier is shown in black, another one in grey. The other two, which stay at +1 , are represented by crosses. 


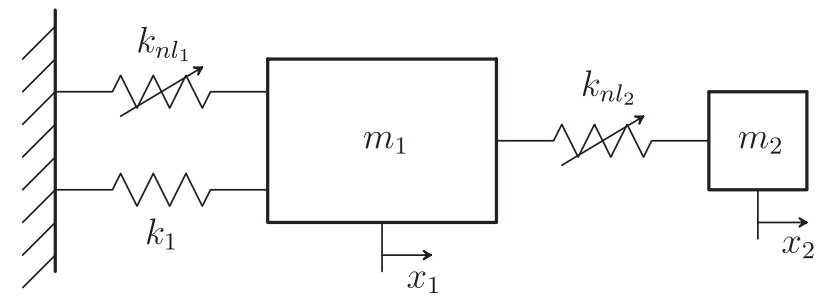

Fig. 17. The $2 \mathrm{DOF}$ system with an essential nonlinearity.

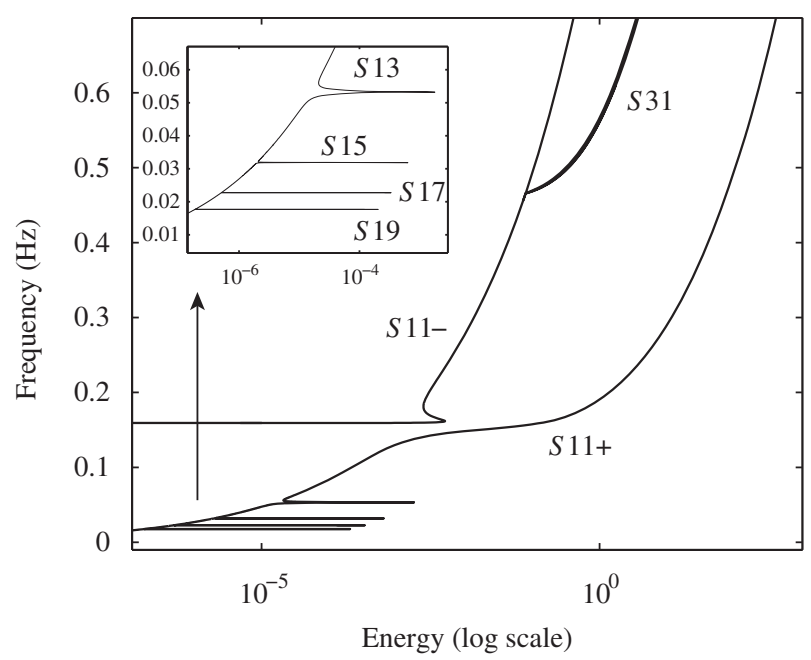

Fig. 18. Frequency-energy plot for the 2DOF system with an essential nonlinearity.

resonant frequency; it can, therefore, interact with an SDOF structure in a frequency-independent fashion or with any mode of an MDOF structure.

For this example and the next two ones, only the practical strategy is utilized. Fig. 18 represents the computed branches, which include $S 11+, S 11-$, and several tongues $(S 13, S 31, S 15, S 17, S 19)$. For clarity, stability is not indicated in this plot. A particular characteristic of this system is that $S 11+$ is not bounded in frequency unlike the previous system.

\subsection{Nonlinear bladed disk system}

The NNMs of a more complex structure, a nonlinear bladed disk, are now investigated. The bladed disk is composed of 20 sectors assembled with cyclic periodicity; a single sector is represented in Fig. 19. Each sector is modeled using disk ( $M$ ) and blade $(m)$ lumped masses, coupled by linear $(k)$ and cubic $\left(k_{n l}\right)$ springs. The nonlinear springs can be representative of geometrically nonlinear effects in the blades. The disk masses are connected together by linear springs $K$. The equations of motion of this 40-DOF system are

$$
\begin{aligned}
& m \ddot{x}_{i}+k\left(x_{i}-X_{i}\right)+k_{n l}\left(x_{i}-X_{i}\right)^{3}=0 \\
& M \ddot{X}_{i}+K\left(X_{i}-X_{i+1}\right)+K\left(X_{i}-X_{i-1}\right)+k\left(X_{i}-x_{i}\right)+k_{n l}\left(X_{i}-x_{i}\right)^{3}=0
\end{aligned}
$$

for $i=1, \ldots, 20 ; X_{21}=X_{1}, X_{0}=X_{20}$ (conditions of cyclic periodicity) and $M=1, m=0.3, K=1, k_{l}=1, k_{n l}=0.5$. $X_{i}$ and $x_{i}$ are the displacements of the disk and blade masses of the $i$ th sector, respectively.

As the structure is not fixed, the first mode is a rigid-body mode, which is obviously not affected by the nonlinearities. Due to periodicity of the structure, several elastic modes of the underlying linear system appear in pair at the same frequencies, with only a spatial shift between them. It is the case of the first two LNMs that possess one nodal diameter. Their nonlinear extensions are represented in the FEP of Fig. 20. The nonlinearities have a slight, but identical (i.e., the backbones cannot be distinguished in the FEP), influence on these two NNMs. Therefore, the continuation can be performed by means of large stepsizes, and only a few seconds are necessary to obtain the backbones for 100 time steps over the halfperiod.

Nonlinear systems with spatial symmetries can possess similar NNMs. This is illustrated in Figs. 21 and 22 for the 21 st NNM of the structure. While its frequency is altered by the nonlinearities in the system, the distribution of the modal shape remains unchanged, which characterizes similar modes. 

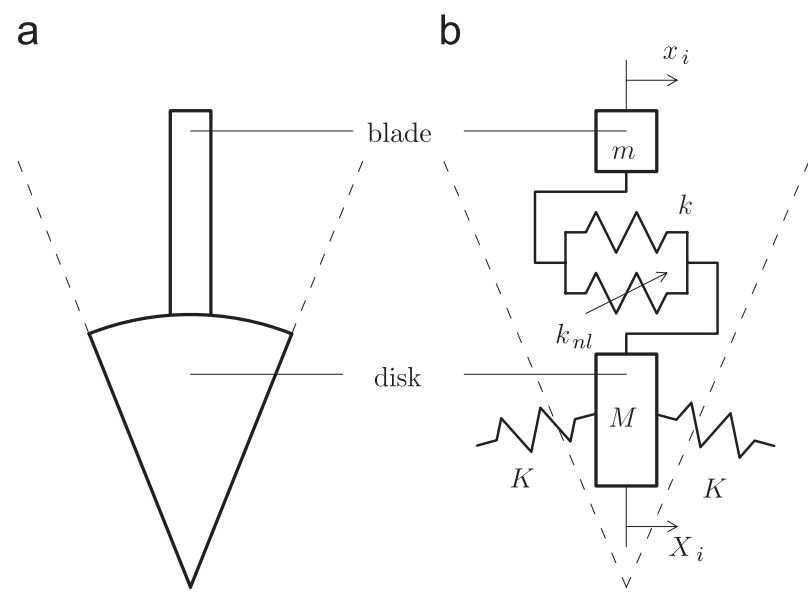

Fig. 19. One sector of the nonlinear bladed disk assembly. (a) Continuous structure; and (b) discrete model.

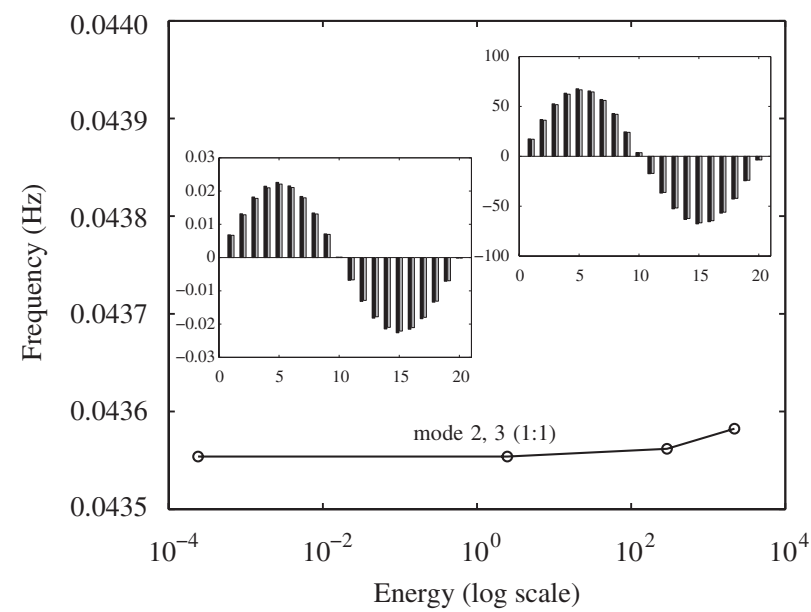

Fig. 20. Frequency-energy plot of the 1 st and 2 nd modes of the bladed disk. The two backbones coincide. The maximum amplitudes of the NNM motions represented by bar graphs are inset; the horizontal axis represents the sector number, and the vertical axis represents the maximum displacements of blade and disk masses (shown in black and grey, respectively).

Finally, a higher mode (i.e., the 38th) for which the corresponding LNM possesses one nodal circle and nine nodal diameters is investigated (see Figs. 23 and 24). Due to the presence of a nodal circle, the blade and disk masses vibrate in an out-of-phase fashion, which enhances the nonlinear effects. As a result, the frequency and the shape of the NNM are strongly affected by the nonlinearities. Furthermore, for increasing energy, there is a clear spatial confinement of vibrational energy to two sectors of the system. Interestingly, this occurs in a perfectly symmetric bladed disk assembly, which has no counterpart in linear theory. We note that the computation of this branch needs approximately 1 min for 100 time steps over the half period.

\subsection{Nonlinear cantilever beam}

As a final example, a planar cantilever beam discretized by 20 finite elements and with a cubic spring at the free end is considered. The geometrical and mechanical properties of the beam are listed in Table 1 . This model is a real nonlinear beam that was used as a benchmark for nonlinear system identification during the European Action COST F3 [37].

The FEP of the first mode and the related NNM motions are plotted in Figs. 25 and 26. The frequency of the NNM motions on the backbone increases with increasing energy levels, which highlights the hardening characteristic of the cubic nonlinearity. The FEP also highlights the presence of one tongue, revealing the existence of a 5:1 internal resonance between the first two modes. When the energy gradually increases along the tongue, a smooth transition from the first to the second mode occurs (see (e) and (f) in Fig. 26). The computation of the backbone branch up to the tongue needs 5 min with 200 time steps over the half-period. Due to the presence of turning points, the computation of the tongue is more expensive and demands 10 min. 


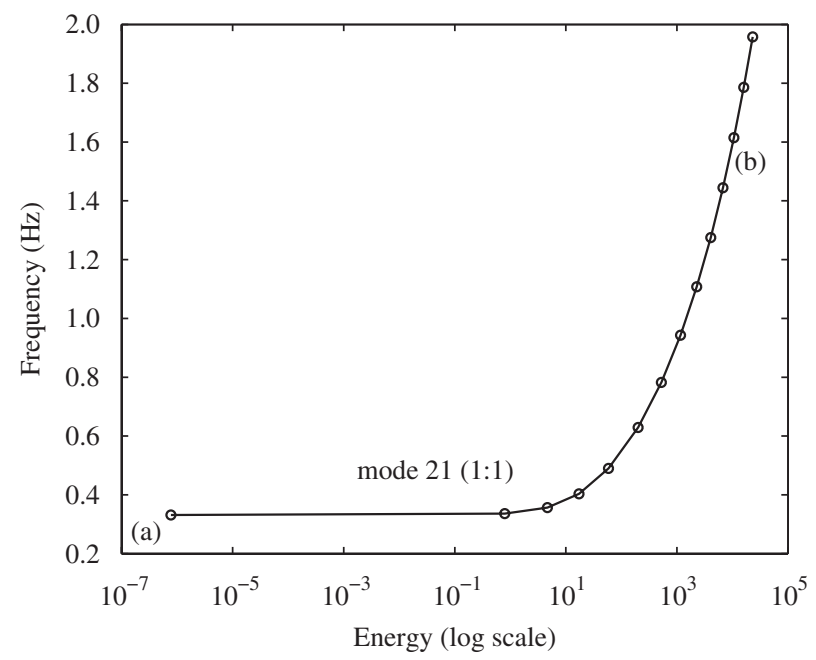

Fig. 21. Frequency-energy plot of the 21 st mode of the nonlinear bladed disk assembly.

a

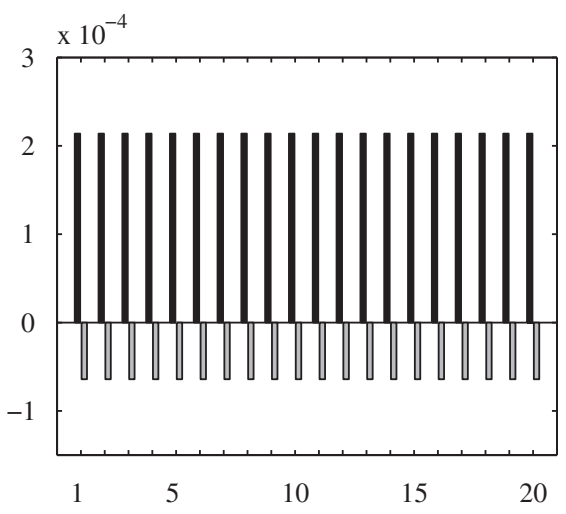

b

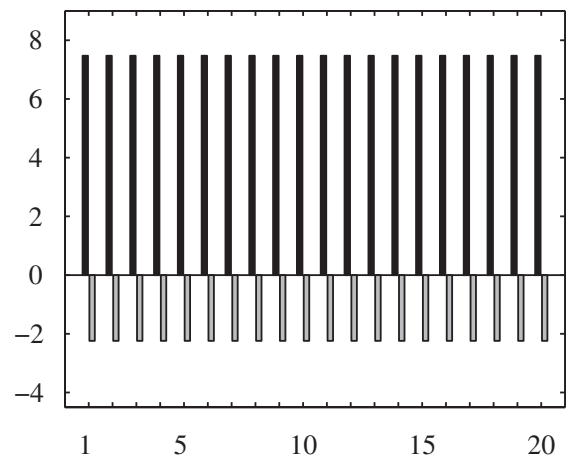

Fig. 22. Maximum amplitudes of the 21st NNM of the bladed disk system at (a) low energy and (b) at high energy. The horizontal axis represents the sector number, and the vertical axis represents the maximum displacements of blade and disk masses (shown in black and grey, respectively).

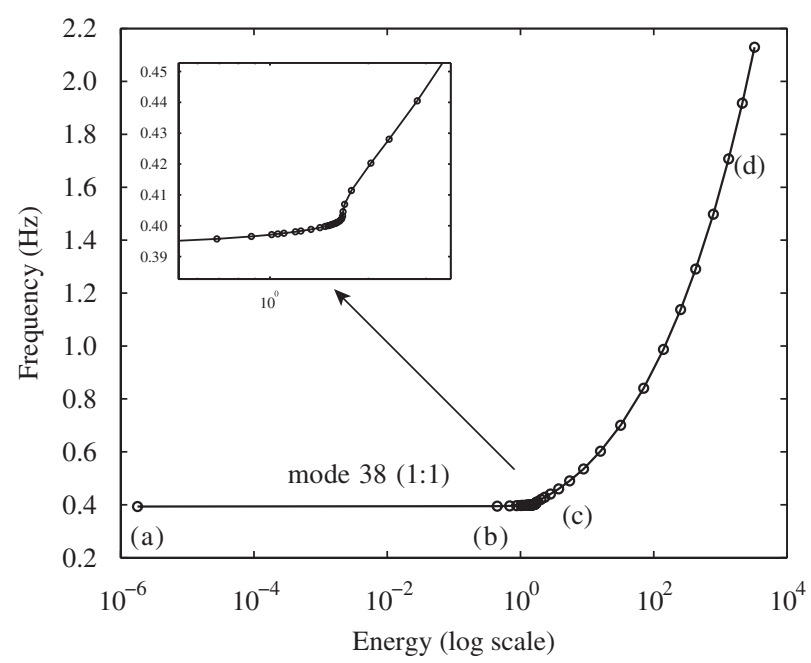

Fig. 23. Frequency-energy plot of the 38th mode of the nonlinear bladed disk assembly. 
a

$$
\mathrm{X} 10^{-4}
$$
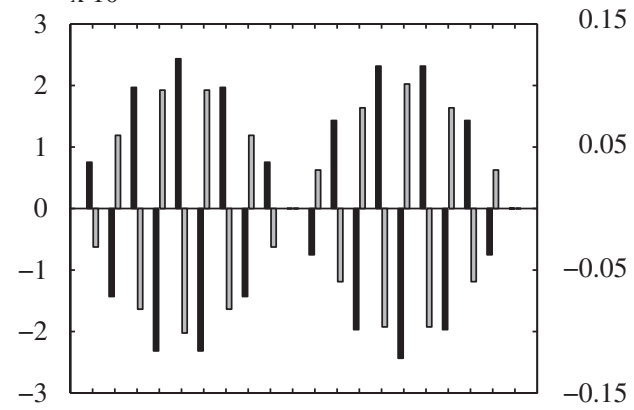

C

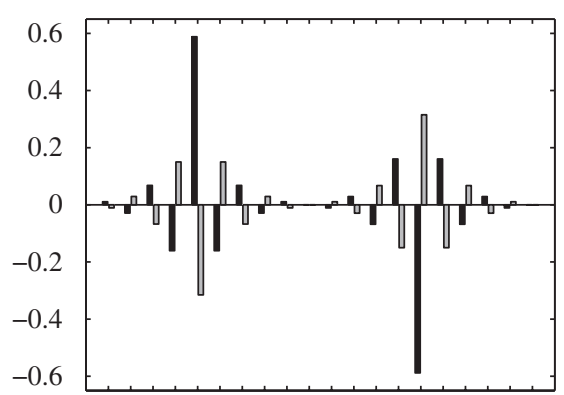

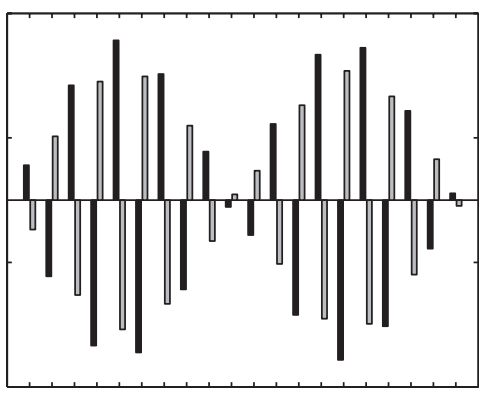

b

d

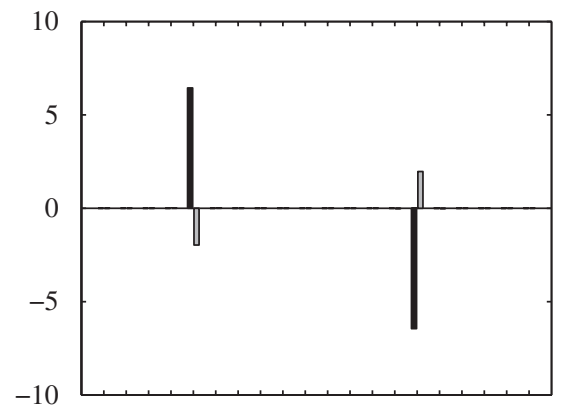

Fig. 24. Maximum amplitudes of the 38th NNM of the bladed disk at (a) low energy, (b) and (c) medium energy and (d) high energy, corresponding to the points in Fig. 23. The horizontal axis represents the sector number, and the vertical axis represents the maximum displacements of blade and disk masses (shown in black and grey, respectively).

Table 1

Geometrical and mechanical properties of the planar cantilever beam

\begin{tabular}{|c|c|c|c|c|c|}
\hline Length ( $\mathrm{m}$ ) & Width (m) & Thickness (m) & Young's modulus $\left(\mathrm{N} / \mathrm{m}^{2}\right)$ & Density $\left(\mathrm{kg} / \mathrm{m}^{3}\right)$ & Nonlinear coeff. $\left(\mathrm{N} / \mathrm{m}^{3}\right)$ \\
\hline 0.7 & 0.014 & 0.014 & $2.05 \mathrm{e} 11$ & 7800 & $6 \times 10^{9}$ \\
\hline
\end{tabular}

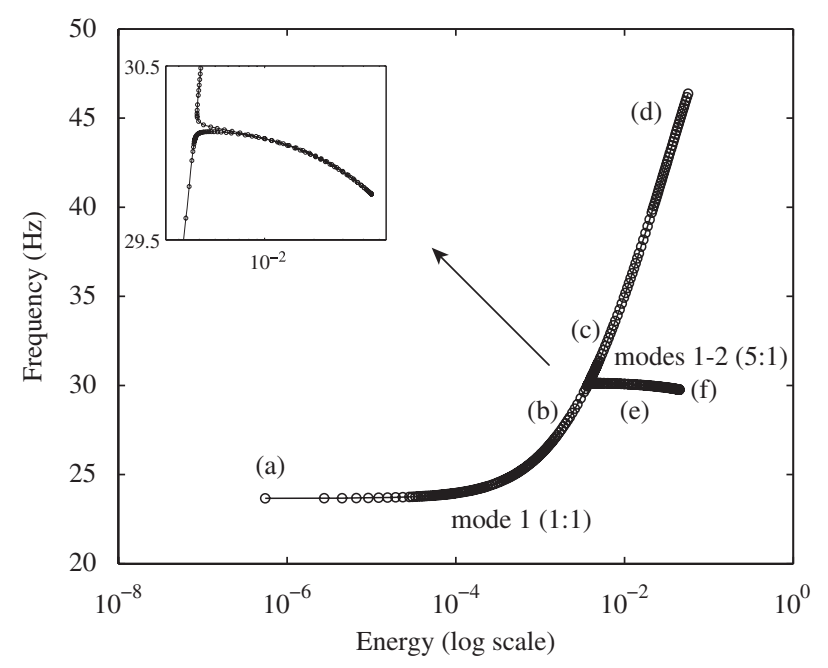

Fig. 25. Frequency-energy plot of the first NNM of the nonlinear cantilever beam. 

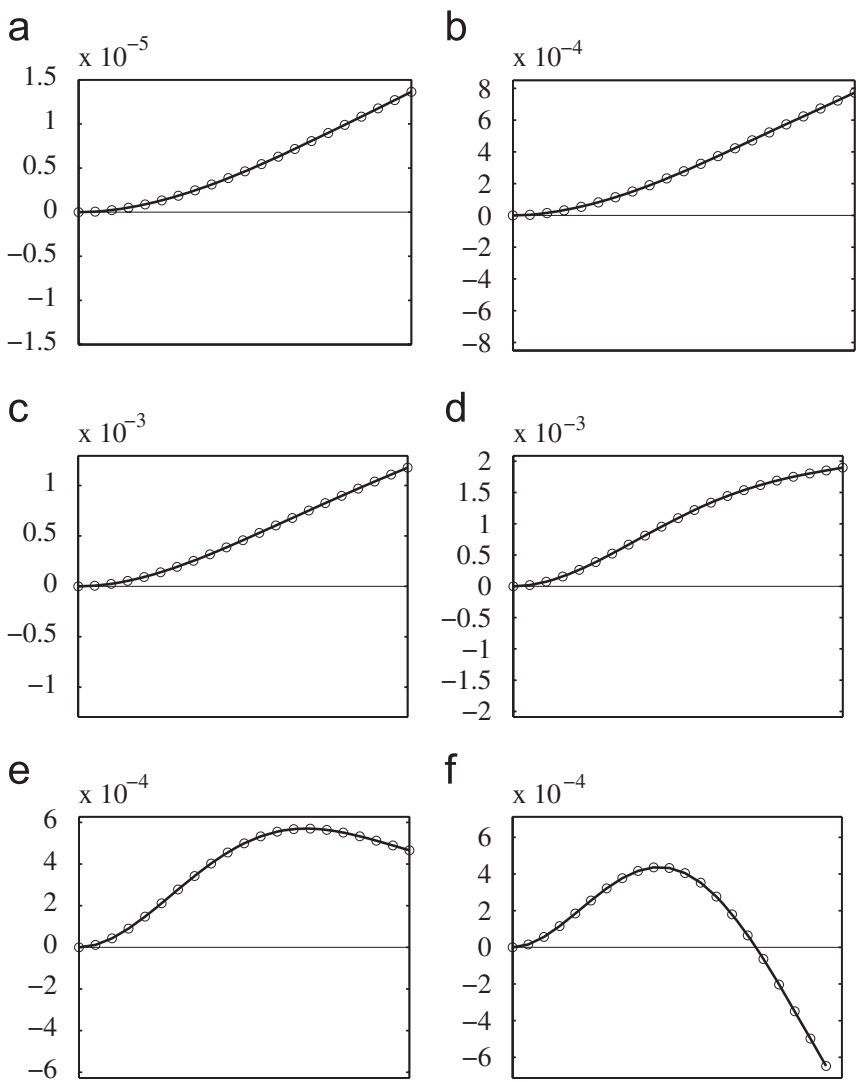

Fig. 26. Maximum amplitudes of the first NNM of the nonlinear beam at different energy levels represented in Fig. 25. (a)-(d): Fundamental (1:1) NNM motions; (e) and (f): internally resonant NNM motions between mode 1 and mode 2.

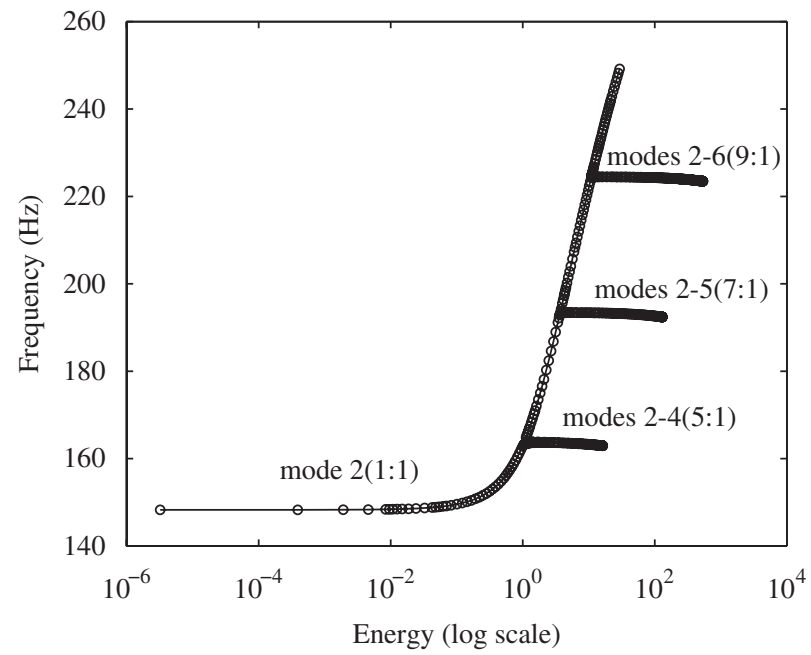

Fig. 27. Frequency-energy plot of the second NNM of the nonlinear cantilever beam.

The second NNM is plotted in the FEP of Fig. 27. Besides the NNM backbone, three tongues are present. The first tongue corresponds to a 5:1 internal resonance between the second and fourth nonlinear modes of the beam. Similarly, a 7:1 internal resonance between the second and fifth modes, and a 9:1 internal resonance between the second and sixth modes are observed.

Similar dynamics were observed for the higher modes and are not further described herein. 


\section{Conclusion and future work}

In this paper, a numerical method for the computation of nonlinear normal modes (NNMs) of nonlinear mechanical structures is introduced. The approach targets the computation of the undamped modes of structures discretized by finite elements and relies on the continuation of periodic solutions. The proposed procedure was demonstrated using different nonlinear structures, and the NNMs were computed accurately in a fairly automatic manner. Complicated NNM motions were also observed, including a countable infinity of internal resonances and strong motion localization.

This method represents a first step toward a practical NNM computation with limited implementation effort. However, two important issues must be addressed adequately to develop a robust method capable of dealing with large, threedimensional structures:

- Fundamental NNMs with no linear counterparts (i.e., those that are not the direct extension of the LNMs) have not been discussed herein. These additional NNMs bifurcate from other modes, and a robust branch switching strategy will be developed for their computation.

- The method relies on extensive numerical simulations and may be computationally intensive for large-scale finite element models. As a result, a further reduction of the computational cost is the next objective. To this end, a significant improvement is to use sensitivity analysis to obtain the Jacobian matrix as a by-product of the time integration of the current motion. An automatic time step control, which selects the most appropriate time step in view of the current dynamics, will also be considered to speed up the computations.

The final objective of this research is to implement the method into an industrial finite element code.

\section{References}

[1] E. Pesheck, Reduced-order modelling of nonlinear structural systems using nonlinear normal modes and invariant manifolds, Ph.D. Thesis, University of Michigan, Ann Arbor, 2000.

[2] H.B. Keller, Numerical Methods in Bifurcation Problems, Springer, Berlin, 1987.

[3] E. Doedel, Auto, software for continuation and bifurcation problems in ordinary differential equations /http://indy.cs.concordia.ca/auto/〉.

[4] R. Seydel, Practical bifurcation and stability analysis. From Equilibrium to Chaos, 2nd ed., Springer, Berlin, 1994.

[5] A.H. Nayfeh, B. Balachandran, Applied Nonlinear Dynamics, Analytical, Computational, and Experimental Methods, Wiley-Interscience, Chichester, 1995.

[6] C. Padmanabhan, R. Singh, Analysis of periodically excited nonlinear-systems by a parametric continuation technique, Journal of Sound and Vibration 184 (1) (1995) 35-58.

[7] P. Sundararajan, S.T. Noah, An algorithm for response and stability of large order non-linear systems - application to rotor systems, Journal of Sound and Vibration 4 (1) (1998) 695-723.

[8] P. Ribeiro, Non-linear forced vibrations of thin/thick beams and plates by the finite element and shooting methods, Computers and Structures 82 (17-19) (2004) 1413-1423.

[9] W. Govaerts, Y.A. Kuznetsov, A. Dhooge, Numerical continuation of bifurcations of limit cycles in MATLAB, SIAM Journal on Scientific Computing 27 (1) (2005) 231-252.

[10] C. Touzé, M. Amabili, Nonlinear normal modes for damped geometrically nonlinear systems: application to reduced-order modelling of harmonically forced structures, Journal of Sound and Vibration 298 (4-5) (2006) 958-981.

[11] C. Touzé, M. Amabili, O. Thomas, Reduced-order models for large-amplitude vibrations of shells including in-plane inertia, in: Proceedings of the EUROMECH Colloquium on Geometrically Nonlinear Vibrations, 2007.

[12] F.J. Munoz-Almaraz, E. Freire, J. Galán, E. Doedel, A. Vanderbauwhede, Continuation of periodic orbits in conservative and hamiltonian systems, Physica D 181 (2003) 1-38.

[13] E.J. Doedel, R.C. Paffenroth, H.B. Keller, D.J. Dickmann, J. Galan Vioque, A. Vanderbauwhede, Computation of periodic solutions of conservative systems with application to the 3-body problem, International Journal of Bifurcations and Chaos 13 (6) (2003) 1-29.

[14] J.C. Slater, A numerical method for determining nonlinear normal modes, Nonlinear Dynamics 10 (1) (1996) 19-30.

[15] Y.S. Lee, G. Kerschen, A.F. Vakakis, P.N. Panagopoulos, L.A. Bergman, D.M. McFarland, Complicated dynamics of a linear oscillator with a light, essentially nonlinear attachment, Physica D 204 (2005) 41-69.

[16] F.X. Wang, A.K. Bajaj, Nonlinear normal modes in multi-mode models of an inertially coupled elastic structure, Nonlinear Dynamics 47 (2007) $25-47$.

[17] R. Arquier, S. Bellizzi, R. Bouc, B. Cochelin, Two methods for the computation of nonlinear modes of vibrating systems at large amplitudes, Computers and Structures 84 (24-25) (2006) 1565-1576.

[18] R.I. Leine, H. Nijmeijer, Dynamics and Bifurcations of Non-smooth Mechanical Systems, Springer, Berlin, 2004.

[19] R.M. Rosenberg, Normal modes of nonlinear dual-mode systems, Journal of Applied Mechanics 27 (1960) 263-268.

[20] R.M. Rosenberg, The normal modes of nonlinear $n$-degree-of-freedom systems, Journal of Applied Mechanics 30 (1) (1962) 7-14.

[21] R.M. Rosenberg, On nonlinear vibrations of systems with many degrees of freedom, Advances in Applied Mechanics 242 (9) (1966) 155-242.

[22] S.W. Shaw, C. Pierre, Nonlinear normal modes and invariant manifolds, Journal of Sound and Vibration 150 (1) (1991) 170-173.

[23] S.W. Shaw, C. Pierre, On nonlinear normal modes, in: ASME Winter Annual Meeting, 1992.

[24] S.W. Shaw, C. Pierre, Normal modes for nonlinear vibratory systems, Journal of Sound and Vibration 164 (1) (1993) 85-124.

[25] S.W. Shaw, C. Pierre, Normal modes of vibration for nonlinear continuous systems, Journal of Sound and Vibration 169 (3) (1994) $319-347$.

[26] G. Kerschen, Y.S. Lee, A.F. Vakakis, D.M. McFarland, L.A. Bergman, Irreversible passive energy transfer in coupled oscillators with essential nonlinearity, SIAM Journal of Applied Mathematics 66 (2) (2006) 648-679.

[27] A.F. Vakakis, L.I. Manevitch, Y.V. Mikhlin, V.N. Pilipchuk, A.A. Zevin, Normal Modes and Localization in Nonlinear Systems, Wiley, New York, 1996.

[28] A.F. Vakakis, R.H. Rand, Normal modes and global dynamics of a 2-degree-of-freedom nonlinear-system; part I: low energies, International Journal of Non-Linear Mechanics 27 (1992) 861-874.

[29] A.F. Vakakis, R.H. Rand, Normal modes and global dynamics of a 2-degree-of-freedom nonlinear-system; part II: high energies, International Journal of Non-Linear Mechanics 27 (1992) 875-888.

[30] T.S. Parker, L.O. Chua, Practical Numerical Algorithms for Chaotic Systems, Springer, New York, 1989.

[31] O. Brüls, P. Eberhard, Sensitivity analysis for dynamic mechanical systems with finite rotations, International Journal for Numerical Methods in Engineering 1 (2006) 1-29. 
[32] N. Mihajlović, Torsional and lateral vibrations in flexible rotor systems with friction, Ph.D. Thesis, Eindhoven University of Technology, 2005.

[33] E.L. Allgower, K. Georg, Introduction to Numerical Continuation Methods, Classics in Applied Mathematics, SIAM, Philadelphia, PA, 2003.

[34] M. Géradin, D. Rixen, Mechanical Vibrations, Theory and Application to Structural Dynamics, Wiley, New York, 1994.

[35] O.V. Gendelman, L.I. Manevitch, A.F. Vakakis, R. McCloskey, Energy pumping in nonlinear mechanical oscillators: part I—dynamics of the underlying hamiltonian systems, Journal of Applied Mechanics 68 (2001) 34-41.

[36] A.F. Vakakis, O.V. Gendelman, Energy pumping in nonlinear mechanical oscillators: part II—resonance capture, Journal of Sound and Vibration 68 (2001) 42-48.

[37] F. Thouverez, Presentation of the ECL benchmark, Mechanical Systems and Signal Processing 17 (2003) 195-202. 